Article

\title{
Green Functions Scattering in the Casimir Effect
}

\author{
Valery N. Marachevsky ${ }^{*},+\left(\mathbb{D}\right.$ and Arseny A. Sidelnikov ${ }^{\dagger}$ \\ St. Petersburg State University, 7/9 Universitetskaya Naberezhnaya, 199034 St. Petersburg, Russia; \\ st074065@student.spbu.ru \\ * Correspondence: maraval@mail.ru or v.marachevsky@spbu.ru \\ + These authors contributed equally to this work.
}

check for

updates

Citation: Marachevsky, V.N.;

Sidelnikov, A.A. Green Functions Scattering in the Casimir Effect.

Universe 2021, 7, 195. https://

doi.org/10.3390/universe7060195

Academic Editors: Galina L.

Klimchitskaya and Gerald B. Cleaver

Received: 29 April 2021

Accepted: 7 June 2021

Published: 9 June 2021

Publisher's Note: MDPI stays neutral with regard to jurisdictional claims in published maps and institutional affiliations.

Copyright: (c) 2021 by the authors. Licensee MDPI, Basel, Switzerland. This article is an open access article distributed under the terms and conditions of the Creative Commons Attribution (CC BY) license (https:// creativecommons.org/licenses/by/ $4.0 /)$.
Abstract: We propose Green functions scattering method to obtain the Casimir-Polder potential between anisotropic atom and one or two planar parallel plates. Lifshitz formula for pressure between two dielectric half-spaces separated by a vacuum slit is derived within the same method. The method is also applied to known conducting systems including graphene which are overviewed.

Keywords: Casimir force; Casimir-Polder potential; anisotropic polarizability; surface conductivity; spatial dispersion; polarization operator; graphene

\section{Introduction}

The Casimir effect [1,2] is a quantum interaction effect between macroscopic objects. Two main ingredients of the effect are geometry of interacting objects and their material properties [3]. Various reviews and books are dedicated to the Casimir effect or related mathematical techniques [4-22]. In this paper we present another derivation of the Casimir effect. One can use this paper as an introduction to the Casimir effect: detailed derivations of classical results are presented, flat boundaries are considered.

The ground state energy of the bosonic system is defined by expression

$$
\tilde{E}=\sum_{i} \frac{\hbar \omega_{i}}{2},
$$

where the sum is over all eigenfrequencies of the system. In quantum field theory the ground state energy (1) needs to be regularized. After subtraction of self-energy terms one obtains the Casimir energy $E$. In the system of two infinite perfectly conducting parallel plates separated by a vacuum slit $d$ the Casimir energy per unit area of the plates was derived in Ref. [2] by application of Euler-Maclaurin formula:

$$
\frac{E}{S}=-\frac{\pi^{2} \hbar c}{720 d^{3}}
$$

here $S$ is the plate area.

Another way of finding the Casimir energy is to use the argument principle

$$
\frac{1}{2 \pi i} \oint \phi(\omega) \frac{d}{d \omega} \ln f(\omega, \beta) d \omega=\sum \phi\left(\omega_{0}\right)-\sum \phi\left(\omega_{\infty}\right)
$$

with

$$
\phi(\omega)=\frac{\hbar \omega}{2}
$$

to evaluate the sum (1). Here $\omega_{0}$ are zeroes and $\omega_{\infty}$ are poles of the function $f(\omega, \beta)$ inside integration contour, degenerate eigenvalues are summed according to their multiplicities. Eigenfrequencies $\omega_{i}$ may also depend on continuous variables $\beta$. The equation for eigenfrequencies is $f\left(\omega_{i}, \beta\right)=0$. This technique was first applied to evaluation of the Casimir 
energy in Ref. [23]. The approach based on Formula (3) was strongly advocated in the review [5]. Fock-Schwinger regularization was applied to derivation of the Casimir result for two perfectly conducting plates (2) in Ref. [24]. Detailed study of the Lifshitz energy [3] for two half-spaces separated by a vacuum slit was performed in Ref. [25] based on the argument principle (3) and plasma model of the permittivity.

The formalism was effectively generalized to non-flat geometries. Theoretical formalism for evaluation of the Casimir free energy and the Casimir force in the system of two diffraction gratings separated by a vacuum slit was developed in Refs. [26,27]. In Refs. [26,27] the Casimir energy was expressed through Rayleigh reflection coefficients [28]. The theory [26] was found to be in agreement with experimental results of Ref. [29] for the normal Casimir force in sphere - rectangular grating geometry. In Refs. [30,31] a comparison of the theory and experiment was performed in the system of two Au sinusoidal gratings with coinciding periods, one of the gratings was skillfully imprinted at the surface of Au sphere. Theory of the lateral Casimir force based on Rayleigh reflection coefficients was found to be in excellent agreement with experiment [30,31], strong deviations from proximity force approximation [32] based on Lifshitz theory for flat geometries were discovered. The Casimir normal force measurements between Au sphere and Si grating [33] and experiments $[34,35]$ with two rectangular gratings separated by a vacuum slit were also found to be in agreement with theoretical approach developed in Refs. [26,27]. Implication of the formalism based on Rayleigh coefficients to evaluation of torque between two gratings separated by a vacuum slit led to theoretical discovery of geometric transitions in Ref. [36]. An abrupt change in the energy of infinite system during an infinitesimal change of rotation angle between gratings discovered in Ref. [36] results in a giant torque in the system of two finite gratings.

Path-integral approach has been used to derive general analytic results for the Casimir free energy of electromagnetic fields in the presence of several compact objects [37,38]. The Casimir energy for systems with cylindrical and spherical objects has been actively studied [39-41].

The Casimir energy of two Chern-Simons layers in vacuum is derived in Refs. [42,43], repulsive Casimir force between Chern-Simons layers in vacuum and Chern-Simons layers at the boundaries of realistic dielectrics is predicted for an interval of parameters of the layers [42-46]. When one inserts chiral medium in a slit between ordinary materials one can also obtain Casimir repulsion [47,48].

One can extract the Casimir-Polder potential of a neutral atom from interaction energy of two dielectrics by rarefication procedure applied to one dielectric [4,22]. In a dilute limit one should sum the Casimir-Polder potential of every atom in the rarefied dielectric to obtain interaction energy of two dielectrics. Please note that one must introduce anisotropic dielectric to extract the Casimir-Polder potential for anisotropic atom or molecule by rarefication procedure, which is definitely more complex procedure.

It is convenient to derive the Casimir-Polder potential of an atom with anisotropic polarizability directly from first principles of quantum field theory. One can use Green functions in the evaluation of the Casimir-Polder potential. The first calculation of this kind was performed in Ref. [1] for the potential of anisotropic atom interacting with a perfectly conducting plane. Direct procedure proved useful for evaluation of the Casimir-Polder potential for non-flat geometries including perfectly conducting wedge and dielectric diffraction grating [49-51]. Casimir repulsion emerges for anisotropic atom above a hole in metal [52] or anisotropic atom above a dielectric grating [53]. The Casimir-Polder potential of an anisotropic atom (molecule) interacting with Chern-Simons layer was derived in Ref. [54]. Charge-parity-violating effects in Casimir-Polder potentials were studied in Ref. [55]. Other approaches to derivation of the Casimir-Polder potential are based on the harmonic oscillator models [56] and interaction of point dipoles [57].

In this paper, we start from evaluation of the Casimir-Polder potential between anisotropic neutral atom and dielectric half-space. We use Weyl formula [58] for the Green function of Helmholtz equation (note that Weyl formula has been used before in derivations 
of the Casimir effect, for example, in Ref. [38]), take vector potentials in a Lorentz gauge and then express the electromagnetic field from a dipole source in terms of electric and magnetic fields. Various boundary conditions considered in the paper are also written in terms of electric and magnetic fields. Thus, the full procedure is clearly gauge-invariant by construction. We demonstrate how to derive the Casimir force between geometries with flat parallel boundaries within the same formalism and derive general formula for the Casimir-Polder potential of anisotropic atom between two half-spaces with parallel boundaries and given boundary conditions.

The paper is organized as follows. We introduce the method based on Green functions scattering in Section 2 during derivation of the Casimir-Polder potential of anisotropic atom in its ground state interacting with a dielectric half-space with frequency dispersion of permittivity $\varepsilon(\omega)$. In Section 3 we apply the method to derive the well-known Lifshitz result for pressure between two dielectric half-spaces separated by a vacuum slit. In Section 4 we derive the Casimir-Polder potential of anisotropic atom between two dielectric half-spaces with parallel boundaries.

The method developed in Sections 2-4 is applied to known systems with $2+1$ dimensional conducting layers (Sections 5 and 6 ) and impedance boundary conditions for metals (Section 7), an overview of results for these systems is given.

In Section 5 we discuss $2+1$ surface conductivity and derive expressions for reflection coefficients in this case. We also comment results obtained for systems with non-diagonal components of conductivity.

Spatial dispersion in a boundary $2+1$ layer is important for applications, we start discussion of this case by writing boundary conditions in Section 6. We explain generalizations which need to be done to write boundary conditions with a spatial dispersion of surface conductivity at the boundary of a dielectric half-space. We introduce $2+1$ polarization operator and express $2+1$ surface conductivity with a spatial dispersion in terms of a polarization operator. Reflection coefficients for a layer of $2+1$ fermions at the boundary of a dielectric half-space are derived and written explicitly in terms of components of polarization operator in several convenient for use forms. Casimir and Casimir-Polder type formulas in Sections 2-4 are valid in this case after substitution of reflection coefficients into respective formulas.

Next we consider $2+1$ Dirac fermions in graphene and overview main implications for layers with graphene at finite temperature in the Casimir effect. Giant temperature Casimir effect for systems with graphene layers is discussed.

In Section 7 we consider impedance boundary conditions and derive reflection coefficients for frequency and spatial dispersion cases.

We put $\hbar=c=k_{B}=1$ and use Heaviside-Lorentz units. We take magnetic permeability $\mu=1$ in materials and work with a frequency dispersion of permittivity $\varepsilon \equiv \varepsilon(\omega)$ in material volumes.

\section{Casimir-Polder Potential of Anisotropic Atom above a Dielectric Half-Space}

In this section, we derive the Casimir-Polder potential of a neutral anisotropic atom in its ground state located at a distance $L$ from a dielectric half-space.

One can substitute Weyl formula [58]

$$
\frac{e^{i \omega\left|\mathbf{r}^{\prime}-\mathbf{r}\right|}}{4 \pi\left|\mathbf{r}^{\prime}-\mathbf{r}\right|}=i \iint \frac{e^{i\left(k_{x}\left(x^{\prime}-x\right)+k_{y}\left(y^{\prime}-y\right)+\sqrt{\omega^{2}-k_{x}^{2}-k_{y}^{2}}\left(z^{\prime}-z\right)\right)}}{2 \sqrt{\omega^{2}-k_{x}^{2}-k_{y}^{2}}} \frac{d k_{x} d k_{y}}{(2 \pi)^{2}},
$$

valid for $z^{\prime}-z>0$, into the solution of equations for vector potentials in a Lorentz gauge

$$
\begin{aligned}
& \left(\Delta+\omega^{2}\right) \varphi(\omega, \mathbf{r})=-\rho(\omega, \mathbf{r}), \\
& \left(\Delta+\omega^{2}\right) \mathbf{A}(\omega, \mathbf{r})=-\mathbf{j}(\omega, \mathbf{r}) .
\end{aligned}
$$


It is convenient to denote $\mathbf{k}_{\|}=\left(k_{x}, k_{y}\right)$. Then electric and magnetic fields propagating downwards from the source at the point $\mathbf{r}^{\prime}=(0,0, L)$ can be represented in the form

$$
\begin{aligned}
& \mathbf{E}(\omega, \mathbf{r})=\int(\rho(\omega, \widetilde{\mathbf{k}}) \widetilde{\mathbf{k}}-\omega \mathbf{j}(\omega, \widetilde{\mathbf{k}})) \frac{e^{i \mathbf{k}_{\|} \cdot \mathbf{r}_{\|}} e^{i k_{z}(L-z)}}{2 k_{z}} \frac{d^{2} \mathbf{k}_{\|}}{(2 \pi)^{2}}, \\
& \mathbf{B}(\omega, \mathbf{r})=\int[\mathbf{j}(\omega, \widetilde{\mathbf{k}}) \times \widetilde{\mathbf{k}}] \frac{e^{i \mathbf{k}_{\|} \cdot \mathbf{r}_{\|}} e^{i k_{z}(L-z)}}{2 k_{z}} \frac{d^{2} \mathbf{k}_{\|}}{(2 \pi)^{2}},
\end{aligned}
$$

where $k_{z}=\sqrt{\omega^{2}-k_{\|}^{2}}, \widetilde{\mathbf{k}}=\left(\mathbf{k}_{\|},-k_{z}\right), \rho(\omega, \widetilde{\mathbf{k}})$ and $\mathbf{j}(\omega, \widetilde{\mathbf{k}})$ are Fourier components of the source current.

Consider propagation of an electromagnetic field from a dipole source at the point $\mathbf{r}^{\prime}=(0,0, L)$ characterized by electric dipole moment $\mathbf{d}$. In this case, components of the four-current density of the dipole source must be written in the form [54]

$$
\begin{aligned}
& \rho(t, \mathbf{r})=-d^{l}(t) \partial_{l} \delta^{3}\left(\mathbf{r}-\mathbf{r}^{\prime}\right), \\
& j^{l}(t, \mathbf{r})=\partial_{t} d^{l}(t) \delta^{3}\left(\mathbf{r}-\mathbf{r}^{\prime}\right) .
\end{aligned}
$$

Please note that four-current density (10), (11) satisfies the continuity equation $\partial_{t} \rho+$ $\operatorname{div} \mathbf{j}=0$. For the point dipole (10), (11) one should substitute

$$
\begin{aligned}
& \rho(\omega, \mathbf{k})=-d^{l} \int \partial_{l} \delta^{3}\left(\mathbf{r}-\mathbf{r}^{\prime}\right) e^{i \mathbf{k} \cdot \mathbf{r}} d^{3} \mathbf{r}=i \mathbf{d} \cdot \mathbf{k} e^{i k_{z} L}, \\
& j^{l}(\omega, \mathbf{k})=-i \omega d^{l} \int \delta^{3}\left(\mathbf{r}-\mathbf{r}^{\prime}\right) e^{i \mathbf{k} \cdot \mathbf{r}} d^{3} \mathbf{r}=-i \omega d^{l} e^{i k_{z} L}
\end{aligned}
$$

to Equations (8) and (9).

Now consider homogeneous dielectric half-space $z \leq 0$ characterized by frequency dispersion of a dielectric permittivity $\varepsilon(\omega)$. For $z \geq 0$ expansions for electric and magnetic fields can be written in the form

$$
\begin{aligned}
\mathbf{E}_{\mathbf{1}}(\omega, \mathbf{r}) & =\int \mathbf{f}\left(\omega, \mathbf{k}_{\|}\right) e^{i \mathbf{k}_{\|} \cdot \mathbf{r}_{\|}} e^{-i k_{z}(z-L)} d^{2} \mathbf{k}_{\|}+\int \mathbf{v}\left(\omega, \mathbf{k}_{\|}\right) e^{i \mathbf{k}_{\|} \cdot \mathbf{r}_{\|}} e^{i k_{z} z} d^{2} \mathbf{k}_{\|}, \\
\mathbf{B}_{\mathbf{1}}(\omega, \mathbf{r}) & =\frac{1}{\omega} \int \mathbf{g}\left(\omega, \mathbf{k}_{\|}\right) e^{i \mathbf{k}_{\|} \cdot \mathbf{r}_{\|}} e^{-i k_{z}(z-L)} d^{2} \mathbf{k}_{\|}+ \\
& \frac{1}{\omega} \int\left(\left[\mathbf{k}_{\|} \times \mathbf{v}\left(\omega, \mathbf{k}_{\|}\right)\right]+k_{z}\left[\mathbf{n} \times \mathbf{v}\left(\omega, \mathbf{k}_{\|}\right)\right]\right) e^{i \mathbf{k}_{\|} \cdot \mathbf{r}_{\|}} e^{i k_{z} z} d^{2} \mathbf{k}_{\|}
\end{aligned}
$$

with vector functions $\mathbf{f}\left(\omega, \mathbf{k}_{\|}\right), \mathbf{g}\left(\omega, \mathbf{k}_{\|}\right), \mathbf{v}\left(\omega, \mathbf{k}_{\|}\right)$. For a dipole source at the point $(0,0, L)$ one substitutes components of the four-current density (12), (13) to electric and magnetic fields (8), (9) and obtains from comparison with first terms in the right-hand side of (14), (15):

$$
\begin{aligned}
\mathbf{f}\left(\omega, \mathbf{k}_{\|}\right) & =\frac{i}{8 \pi^{2} k_{z}}\left(\left(-\mathbf{d}_{\|} \mathbf{k}_{\|}+d_{z} k_{z}\right) \widetilde{\mathbf{k}}+\omega^{2} \mathbf{d}\right), \\
\mathbf{g}\left(\omega, \mathbf{k}_{\|}\right) & =\frac{i}{8 \pi^{2}} \frac{\omega^{2}}{k_{z}}[\widetilde{\mathbf{k}} \times \mathbf{d}] .
\end{aligned}
$$

For $z \leq 0$ one can write expansions for electric and magnetic fields in the form

$$
\begin{aligned}
& \mathbf{E}_{2}(\omega, \mathbf{r})=\int \mathbf{u}\left(\omega, \mathbf{k}_{\|}\right) e^{i \mathbf{k}_{\|} \cdot \mathbf{r}_{\|}} e^{-i K_{z} z} d^{2} \mathbf{k}_{\|} \\
& \mathbf{B}_{2}(\omega, \mathbf{r})=\frac{1}{\omega} \int\left(\left[\mathbf{k}_{\|} \times \mathbf{u}\left(\omega, \mathbf{k}_{\|}\right)\right]-K_{z}\left[\mathbf{n} \times \mathbf{u}\left(\omega, \mathbf{k}_{\|}\right)\right]\right) e^{i \mathbf{k}_{\|} \cdot \mathbf{r}_{\|}} e^{-i K_{z} z} d^{2} \mathbf{k}_{\|}
\end{aligned}
$$


with $K_{z}=\sqrt{\varepsilon(\omega) \omega^{2}-k_{x}^{2}-k_{y}^{2}}$ and vector function $\mathbf{u}\left(\omega, \mathbf{k}_{\|}\right)$. Functions $\mathbf{v}\left(\omega, \mathbf{k}_{\|}\right)$and $\mathbf{u}\left(\omega, \mathbf{k}_{\|}\right)$are expressed in terms of functions $\mathbf{f}\left(\omega, \mathbf{k}_{\|}\right), \mathbf{g}\left(\omega, \mathbf{k}_{\|}\right)$after imposing boundary conditions and solution of diffraction problem.

Without loss of generality, we may use polar coordinates in two dimensional $\left(k_{x}, k_{y}\right)$ momentum space and orthogonal local basis $\mathbf{e}_{r}, \mathbf{e}_{\theta}, \mathbf{e}_{z}$ so that $\mathbf{k}_{\|}=k_{r} \mathbf{e}_{r}, k_{r}=\left|\mathbf{k}_{\|}\right|$. Reflected and transmitted electric fields are transverse (note that in the local basis $\mathbf{v}\left(\omega, \mathbf{k}_{\|}\right) \mathbf{k}_{\|}=v_{r} k_{r}$, $\left.\mathbf{u}\left(\omega, \mathbf{k}_{\|}\right) \mathbf{k}_{\|}=u_{r} k_{r}\right)$

$$
\begin{aligned}
v_{r} k_{r}+k_{z} v_{z} & =0, \\
u_{r} k_{r}-K_{z} u_{z} & =0 .
\end{aligned}
$$

Continuity of tangential components of the fields at the boundary of dielectric halfspace $z=0$ yields four conditions:

$$
\begin{aligned}
u_{r} & =v_{r}+f_{r} e^{i k_{z} L}, \\
u_{\theta} & =v_{\theta}+f_{\theta} e^{i k_{z} L}, \\
K_{z} u_{\theta} & =-k_{z} v_{\theta}+g_{r} e^{i k_{z} L}, \\
-k_{r} u_{z}-K_{z} u_{r} & =-k_{r} v_{z}+k_{z} v_{r}+g_{\theta} e^{i k_{z} L} .
\end{aligned}
$$

Reflected and transmitted fields are expressed in terms of $f_{r}, f_{\theta}, g_{r}, g_{\theta}$ :

$$
\begin{aligned}
& v_{r}=-e^{i k_{z} L} \frac{k_{z}}{\omega^{2}\left(K_{z}+\varepsilon k_{z}\right)}\left(K_{z} g_{\theta}+\varepsilon \omega^{2} f_{r}\right), \\
& v_{\theta}=e^{i k_{z} L} \frac{1}{k_{z}+K_{z}}\left(g_{r}-f_{\theta} K_{z}\right), \\
& v_{z}=e^{i k_{z} L} \frac{k_{r}}{\omega^{2}\left(K_{z}+\varepsilon k_{z}\right)}\left(K_{z} g_{\theta}+\varepsilon \omega^{2} f_{r}\right), \\
& u_{r}=e^{i k_{z} L} \frac{K_{z}}{\omega^{2}\left(K_{z}+\varepsilon k_{z}\right)}\left(-k_{z} g_{\theta}+\omega^{2} f_{r}\right), \\
& u_{\theta}=e^{i k_{z} L} \frac{1}{k_{z}+K_{z}}\left(g_{r}+f_{\theta} k_{z}\right), \\
& u_{z}=e^{i k_{z} L} \frac{k_{r}}{\omega^{2}\left(K_{z}+\varepsilon k_{z}\right)}\left(-k_{z} g_{\theta}+\omega^{2} f_{r}\right) .
\end{aligned}
$$

In general, $v_{i}=P_{i j} d_{j}$. Non-diagonal components of the matrix $P_{i j}$ do not contribute to the Casimir-Polder potential of a neutral atom interacting with a dielectric half-space. Diagonal components of the matrix $P_{i j}$ can be found from (16), (17) and (26)-(28):

$$
\begin{aligned}
& P_{r r}\left(\omega, k_{r}\right)=-\frac{i}{8 \pi^{2}} e^{i k_{z} L} k_{z} r_{T M}\left(\omega, k_{r}\right), \\
& P_{\theta \theta}\left(\omega, k_{r}\right)=\frac{i}{8 \pi^{2}} e^{i k_{z} L} \frac{\omega^{2}}{k_{z}} r_{T E}\left(\omega, k_{r}\right), \\
& P_{z z}\left(\omega, k_{r}\right)=\frac{i}{8 \pi^{2}} e^{i k_{z} L} \frac{k_{r}^{2}}{k_{z}} r_{T M}\left(\omega, k_{r}\right),
\end{aligned}
$$

where $r_{T M}\left(\omega, k_{r}\right)$ and $r_{T E}\left(\omega, k_{r}\right)$ are Fresnel reflection coefficients

$$
r_{T M}\left(\omega, k_{r}\right)=\frac{\varepsilon k_{z}-K_{z}}{\varepsilon k_{z}+K_{z}}, \quad r_{T E}\left(\omega, k_{r}\right)=\frac{k_{z}-K_{z}}{k_{z}+K_{z}} .
$$


At this point it is natural to define atomic polarizability $\alpha_{i j}(\omega)$ following definitions from Ref. [59]:

$$
\begin{aligned}
& \alpha_{i j}\left(t_{1}-t_{2}\right)=i\left\langle T\left(\hat{d}_{i}\left(t_{1}\right), \hat{d}_{j}\left(t_{2}\right)\right)\right\rangle, \\
& \alpha_{i j}(t)=\int_{-\infty}^{\infty} \exp (-i \omega t) \alpha_{i j}(\omega) \frac{d \omega}{2 \pi},
\end{aligned}
$$

where $\hat{d}_{i}(t)$ in (36) are operators of electric dipole moment, $T$ indicates that the expression is to be evaluated by time-ordering. Additionally, we use relation

$$
D_{i j}\left(t-t^{\prime}, \mathbf{r}, \mathbf{r}^{\prime}\right)=i\left\langle T\left(\hat{E}_{i}(t, \mathbf{r}) \hat{E}_{j}\left(t^{\prime}, \mathbf{r}^{\prime}\right)\right)\right\rangle \text {. }
$$

To find the Casimir-Polder potential at zero temperature one can use definition for the change of the ground state energy level $\Delta E_{0}$ after actuation of the interaction $\hat{V}$ in the Hamiltonian $H=H_{0}+\hat{V}$ [60]:

$$
\langle 0|\hat{S}| 0\rangle=e^{-i \Delta E_{0} \tau}, \quad \tau \rightarrow+\infty,
$$

here $\tau=\tau_{1}-\tau_{2}$ is the time interval which enters standard definition of $\hat{S}\left(\tau_{1}, \tau_{2}\right)$, $\hat{S}$-matrix is defined in the interaction representation [61]:

$$
\begin{aligned}
& \hat{S}\left(\tau_{1}, \tau_{2}\right) \equiv \exp \left(i H_{0} \tau_{1}\right) \exp \left(-i H\left(\tau_{1}-\tau_{2}\right)\right) \exp \left(-i H_{0} \tau_{2}\right), \\
& \hat{S} \equiv \hat{S}(+\infty,-\infty)=T \exp \left(-i \int_{-\infty}^{+\infty} d t \hat{V}(t)\right) .
\end{aligned}
$$

The vacuum element (39) includes disconnected diagrams. We take

$$
\hat{V}(t)=\int d^{3} \mathbf{r} \hat{J}_{\mu}(t, \mathbf{r}) \hat{A}^{\mu}(t, \mathbf{r})
$$

with operators $\hat{d}^{l}(t)$ in the four-current density (10), (11) and obtain

$$
\begin{aligned}
\int d t \hat{V}(t)= & \int d t d^{3} \mathbf{r}\left(\hat{A}_{l}(t, \mathbf{r}) \partial_{t} \hat{d}^{l}(t) \delta^{3}\left(\mathbf{r}-\mathbf{r}^{\prime}\right)-\hat{A}_{0}(t, \mathbf{r}) \hat{d}^{l}(t) \partial_{l} \delta^{3}\left(\mathbf{r}-\mathbf{r}^{\prime}\right)\right)= \\
& \int d t d^{3} \mathbf{r}\left(\left(\partial_{l} \hat{A}_{0}(t, \mathbf{r})-\partial_{0} \hat{A}_{l}(t, \mathbf{r})\right)\right) \hat{d}^{l}(t) \delta^{3}\left(\mathbf{r}-\mathbf{r}^{\prime}\right)=-\int d t \hat{\mathbf{E}}\left(t, \mathbf{r}^{\prime}\right) \hat{\mathbf{d}}(t) .
\end{aligned}
$$

In the second order perturbation theory we use (41)-(43), definitions of atomic polarizability (36), (37) and electric field propagator (38) to obtain

$$
\begin{aligned}
\left\langle 0\left|\hat{S}^{(2)}\right| 0\right\rangle & =\left.\frac{1}{2} \iint d t d t^{\prime} \alpha^{i j}\left(t-t^{\prime}\right)\left(D_{i j}\left(t-t^{\prime}, \mathbf{r}, \mathbf{r}^{\prime}\right)-D_{i j}^{(0)}\left(t-t^{\prime}, \mathbf{r}, \mathbf{r}^{\prime}\right)\right)\right|_{\mathbf{r}^{\prime} \rightarrow \mathbf{r}}= \\
& \frac{\tau}{2} \int_{-\infty}^{+\infty} \frac{d \omega}{2 \pi} \alpha^{i j}(\omega) D_{i j}^{E}\left(|\omega|, \mathbf{r}^{\prime}, \mathbf{r}^{\prime}\right)=i \tau \int_{0}^{+\infty} \frac{d \omega}{2 \pi} \alpha^{i j}(i \omega) D_{i j}^{E}\left(i \omega, \mathbf{r}^{\prime}, \mathbf{r}^{\prime}\right) .
\end{aligned}
$$

In the last equality of (44) we use $\alpha_{i k}(\omega)=\alpha_{k i}(-\omega)$ [62] and make Wick rotation afterwards. When one evaluates the Casimir-Polder potential of a neutral atom in the presence of a dielectric half-space one should use the difference between the full propagator with dielectric half-space and the vacuum propagator at the position of the atom outside dielectric. This calculation leads to the finite value of the Casimir-Polder potential. As a result, one always needs the scattering part of the full propagator to determine the Casimir-Polder potential. The scattering part of the electric field propagator is defined by

$$
D_{i j}^{E}\left(t-t^{\prime}, \mathbf{r}, \mathbf{r}^{\prime}\right)=D_{i j}\left(t-t^{\prime}, \mathbf{r}, \mathbf{r}^{\prime}\right)-D_{i j}^{(0)}\left(t-t^{\prime}, \mathbf{r}, \mathbf{r}^{\prime}\right) .
$$


From (39) and (44) the Casimir-Polder potential is defined in terms of scattering electric Green function (45):

$$
U(L)=-\int_{0}^{\infty} \frac{d \omega}{2 \pi} \alpha^{i j}(i \omega) D_{i j}^{E}\left(i \omega, \mathbf{r}^{\prime}, \mathbf{r}^{\prime}\right) .
$$

Diagonal components of the scattering electric field propagator can be immediately written from (14) and (32)-(34):

$$
\begin{aligned}
& D_{x x}^{E}\left(\omega, \mathbf{r}^{\prime}, \mathbf{r}^{\prime}\right)=\int e^{i k_{z} L}\left(P_{r r}\left(\omega, k_{r}\right) \cos ^{2} \theta+P_{\theta \theta}\left(\omega, k_{r}\right) \sin ^{2} \theta\right) d^{2} \mathbf{k}_{\|}, \\
& D_{y y}^{E}\left(\omega, \mathbf{r}^{\prime}, \mathbf{r}^{\prime}\right)=\int e^{i k_{z} L}\left(P_{r r}\left(\omega, k_{r}\right) \sin ^{2} \theta+P_{\theta \theta}\left(\omega, k_{r}\right) \cos ^{2} \theta\right) d^{2} \mathbf{k}_{\|}, \\
& D_{z z}^{E}\left(\omega, \mathbf{r}^{\prime}, \mathbf{r}^{\prime}\right)=\int e^{i k_{z} L} P_{z z}\left(\omega, k_{r}\right) d^{2} \mathbf{k}_{\|} .
\end{aligned}
$$

Please note that in expressions (47)-(49) we put $\mathbf{r}^{\prime}=(0,0, L)$. We substitute expressions (47)-(49) into a general Formula (46) and find the Casimir-Polder potential of a neutral polarizable atom separated by a distance $L$ from a dielectric half-space [15]:

$$
\begin{aligned}
U(L)=-\int_{0}^{\infty} \frac{d \omega}{2 \pi} \int_{0}^{\infty} \frac{d k_{r} k_{r}}{2 \pi} \frac{\exp \left(-2 \sqrt{\omega^{2}+k_{r}^{2}} L\right)}{2 \sqrt{\omega^{2}+k_{r}^{2}}}\left[r_{T M}\left(i \omega, k_{r}\right) k_{r}^{2} \alpha_{z z}(i \omega)+\right. \\
\\
\left.\frac{1}{2}\left(r_{T M}\left(i \omega, k_{r}\right)\left(\omega^{2}+k_{r}^{2}\right)-r_{T E}\left(i \omega, k_{r}\right) \omega^{2}\right)\left(\alpha_{x x}(i \omega)+\alpha_{y y}(i \omega)\right)\right] .
\end{aligned}
$$

In this paper, we do not construct electric Green functions on the basis of existing transverse electric (TE) and transverse magnetic (TM) mode solutions as it is usually done [63]. In the method developed in this paper TE and TM reflection coefficients appear in solutions of electric Green functions (47)-(49) on the fly from the system of Equations (20)-(25) resulting from transversality conditions and boundary conditions imposed on electric and magnetic fields.

The primary goal of our paper is to illustrate the method on several examples which are overviewed at the same time.

\section{Force between Two Dielectric Half-Spaces}

Consider two dielectric half-spaces $z \leq 0, z \geq d$ and the vacuum slit $0<z<d$ between them. We evaluate the Casimir force and the Casimir-Polder potential of a neutral atom in this system.

Consider propagation of an electromagnetic field from a point dipole located at $\mathbf{r}^{\prime}=\left(0,0, z_{0}\right), z_{0}<d$. Electric and magnetic fields propagating upwards from the dipole source can be represented in the form

$$
\begin{aligned}
& \mathbf{E}=\int \mathbf{N} e^{i \mathbf{k}_{\|} \cdot \mathbf{r}_{\|}} e^{i k_{z}\left(z-z_{0}\right)} d^{2} \mathbf{k}_{\|}, \\
& \mathbf{B}=\frac{1}{\omega} \int[\mathbf{k} \times \mathbf{N}] e^{i \mathbf{k}_{\|} \cdot \mathbf{r}_{\|}} e^{i k_{z}\left(z-z_{0}\right)} d^{2} \mathbf{k}_{\|}=\frac{1}{\omega} \int \mathbf{M} e^{i \mathbf{k}_{\|} \cdot \mathbf{r}_{\|}} e^{i k_{z}\left(z-z_{0}\right)} d^{2} \mathbf{k}_{\|},
\end{aligned}
$$

where $\mathbf{N}=-i\left((\mathbf{k} \cdot \mathbf{d}) \mathbf{k}-\omega^{2} \mathbf{d}\right) /\left(8 \pi^{2} k_{z}\right), \mathbf{M}=[\mathbf{k} \times \mathbf{N}]$. For $z \leq d$ electric and magnetic fields reflected from a boundary $z=d$ have the form

$$
\begin{aligned}
& \mathbf{E}^{1 R}(\omega, \mathbf{r})=\int \mathbf{v}_{\mathbf{1}} e^{i \mathbf{k}_{\|} \cdot \mathbf{r}_{\|}} e^{-i k_{z} z} d^{2} \mathbf{k}_{\|}, \\
& \mathbf{B}^{1 R}(\omega, \mathbf{r})=\frac{1}{\omega} \int\left(\left[\mathbf{k}_{\|} \times \mathbf{v}_{\mathbf{1}}\right]-k_{z}\left[\mathbf{n} \times \mathbf{v}_{\mathbf{1}}\right]\right) e^{i \mathbf{k}_{\|} \cdot \mathbf{r}_{\|}} e^{-i k_{z} z} d^{2} \mathbf{k}_{\|},
\end{aligned}
$$


where we assume that vector function $\mathbf{v}_{\mathbf{1}}$ depends on $\omega, \mathbf{k}_{\|}$, external geometric parameters of the system $z_{0}, d$ and the dipole moment $\mathbf{d}$. Transmitted electric and magnetic fields for $z \geq d$ have the form

$$
\begin{aligned}
& \mathbf{E}^{1 T}(\omega, \mathbf{r})=\int \mathbf{u}_{\mathbf{1}} e^{i \mathbf{k}_{\|} \cdot \mathbf{r}_{\|}} e^{i K_{z 1} z} d^{2} \mathbf{k}_{\|}, \\
& \mathbf{B}^{1 T}(\omega, \mathbf{r})=\frac{1}{\omega} \int\left(\left[\mathbf{k}_{\|} \times \mathbf{u}_{\mathbf{1}}\right]+K_{z 1}\left[\mathbf{n} \times \mathbf{u}_{\mathbf{1}}\right]\right) e^{i \mathbf{k}_{\|} \cdot \mathbf{r}_{\|}} e^{i K_{z 1} z} d^{2} \mathbf{k}_{\|} .
\end{aligned}
$$

One may use the local basis $\mathbf{e}_{r}, \mathbf{e}_{\theta}, \mathbf{e}_{z}$ for every $\mathbf{k}_{\|}$to find local contributions to the components of the fields. Electric field is transverse:

$$
\begin{aligned}
u_{1 r} k_{r}+K_{z 1} u_{1 z} & =0, \\
v_{1 r} k_{r}-k_{z} v_{1 z} & =0 .
\end{aligned}
$$

Tangential components of electric and magnetic fields are continuous at $z=d$, which yields four conditions:

$$
\begin{aligned}
u_{1 r} e^{i K_{z 1} d} & =v_{1 r} e^{-i k_{z} d}+N_{r} e^{i k_{z}\left(d-z_{0}\right)}, \\
u_{1 \theta} e^{i K_{z 1} d} & =v_{1 \theta} e^{-i k_{z} d}+N_{\theta} e^{i k_{z}\left(d-z_{0}\right)} \\
-K_{z 1} u_{1 \theta} e^{i K_{z 1} d} & =k_{z} v_{1 \theta} e^{-i k_{z} d}+M_{r} e^{i k_{z}\left(d-z_{0}\right)}, \\
-k_{r} u_{1 z} e^{i K_{z 1} d}+K_{z 1} u_{1 r} e^{i K_{z 1} d} & =-k_{r} v_{1 z} e^{-i k_{z} d}-k_{z} v_{1 r} e^{-i k_{z} d}+M_{\theta} e^{i k_{z}\left(d-z_{0}\right)} .
\end{aligned}
$$

One can express reflected fields in terms of $N_{r}, N_{\theta}, M_{r}, M_{\theta}$ :

$$
\begin{aligned}
& v_{1 r}=\frac{k_{z} e^{i k_{z}\left(2 d-z_{0}\right)}}{\omega^{2}\left(\varepsilon k_{z}+K_{z 1}\right)}\left(K_{z 1} M_{\theta}-\varepsilon \omega^{2} N_{r}\right), \\
& v_{1 \theta}=-\frac{e^{i k_{z}\left(2 d-z_{0}\right)}}{k_{z}+K_{z 1}}\left(M_{r}+N_{\theta} K_{z 1}\right), \\
& v_{1 z}=\frac{k_{r} e^{i k_{z}\left(2 d-z_{0}\right)}}{\omega^{2}\left(\varepsilon k_{z}+K_{z 1}\right)}\left(K_{z 1} M_{\theta}-\varepsilon \omega^{2} N_{r}\right),
\end{aligned}
$$

where

$$
\begin{aligned}
& N_{r}=-\frac{i}{8 \pi^{2}}\left(k_{r} d_{z}-k_{z} d_{r}\right), \\
& N_{\theta}=\frac{i}{8 \pi^{2}} \frac{\omega^{2}}{k_{z}} d_{\theta}, \\
& N_{z}=-\frac{k_{r}}{k_{z}} N_{r} .
\end{aligned}
$$

Finally, one finds:

$$
\begin{aligned}
& v_{1 r}=-N_{r} r_{T M 1} e^{i k_{z}\left(2 d-z_{0}\right),} \\
& v_{1 \theta}=N_{\theta} r_{T E 1} e^{i k_{z}\left(2 d-z_{0}\right)}, \\
& v_{1 z}=N_{z} r_{T M 1} e^{i k_{z}\left(2 d-z_{0}\right)} .
\end{aligned}
$$


To obtain the components of reflected fields $v_{x}, v_{y}$ one should use rotation between two local bases and make substitutions for every given $\mathbf{k}_{\|}$in expression (53) for the reflected field:

$$
\begin{aligned}
d_{r} & =d_{x} \cos \theta+d_{y} \sin \theta, \\
d_{\theta} & =d_{x} \sin \theta-d_{y} \cos \theta, \\
v_{1 x} & =v_{1 r} \cos \theta+v_{1 \theta} \sin \theta, \\
v_{1 y} & =v_{1 r} \sin \theta-v_{1 \theta} \cos \theta .
\end{aligned}
$$

Therefore, the electric field reflected from a boundary $z=d$ can be written in the form (53) with vector functions (69)-(71) in the basis $\mathbf{e}_{r}, \mathbf{e}_{\theta}, \mathbf{e}_{z}$. Reflected and transmitted electric fields after diffraction at the boundary $z=0$ can be written as

$$
\begin{aligned}
& \mathbf{E}^{2 R}=\int \mathbf{v}_{2} e^{i \mathbf{k}_{\|} \cdot \mathbf{r}_{\|}} e^{i k_{z} z} d^{2} \mathbf{k}_{\|}, \\
& \mathbf{E}^{2 T}=\int \mathbf{u}_{2} e^{i \mathbf{k}_{\|} \cdot \mathbf{r}_{\|}} e^{-i K_{z 2} z} d^{2} \mathbf{k}_{\|},
\end{aligned}
$$

where now one finds

$$
\begin{aligned}
& v_{2 r}=-v_{1 r} r_{T M 2}=N_{r} r_{T M 1} r_{T M 2} e^{i k_{z}\left(2 d-z_{0}\right)}, \\
& v_{2 \theta}=v_{1 \theta} r_{T E 2}=N_{\theta} r_{T E 1} r_{T E 2} e^{i k_{z}\left(2 d-z_{0}\right)}, \\
& v_{2 z}=v_{1 z} r_{T M 2}=N_{z} r_{T M 1} r_{T M 2} e^{i k_{z}\left(2 d-z_{0}\right)} .
\end{aligned}
$$

If one continues along the same lines then, for example, after $n$ reflections from $z=d$ boundary

$$
v_{n r}=-N_{r} e^{i k_{z}\left(2 n d-z_{0}\right)} r_{T M 1}^{n} r_{T M 2}^{n-1},
$$

and for consecutive reflection from $z=0$ boundary

$$
v_{(n+1) r}=N_{r} e^{i k_{z}\left(2 n d-z_{0}\right)} r_{T M 1}^{n} r_{T M 2}^{n} .
$$

After summation of all reflections of the electric field which first reflects from $z=d$ boundary one obtains

$$
\begin{aligned}
\mathbf{E}_{u p}(\mathbf{r})=\int d^{2} \mathbf{k}_{\|} e^{i \mathbf{k}_{\|} \cdot \mathbf{r}_{\|}} \frac{e^{i k_{z}\left(2 d-z_{0}\right)}}{1-r_{T M 1} r_{T M 2} e^{2 i k_{z} d}}\left[\mathbf{e}_{r} N_{r}\left(r_{T M 1} r_{T M 2} e^{i k_{z} z}-r_{T M 1} e^{-i k_{z} z}\right)+\right. \\
\left.\mathbf{e}_{\theta} N_{\theta}\left(r_{T E 1} r_{T E 2} e^{i k_{z} z}+r_{T E 1} e^{-i k_{z} z}\right)+\mathbf{e}_{z} N_{z}\left(r_{T M 1} r_{T M 2} e^{i k_{z} z}+r_{T M 1} e^{-i k_{z} z}\right)\right] .
\end{aligned}
$$

In full analogy, after summation of all reflections of the electric field which first reflects from $z=0$ boundary one derives

$$
\begin{aligned}
\mathbf{E}_{\text {down }}(\mathbf{r})= & \int d^{2} \mathbf{k}_{\|} e^{i \mathbf{k}_{\|} \cdot \mathbf{r}_{\|}} \frac{e^{i k_{z}\left(2 d+z_{0}\right)}}{1-r_{T M 1} r_{T M 2} e^{2 i k_{z} d}}\left[\mathbf{e}_{r} \widetilde{N}_{r}\left(r_{T M 1} r_{T M 2} e^{-i k_{z} z}-r_{T M 2} e^{i k_{z} z}\right)+\right. \\
& \left.\mathbf{e}_{\theta} \widetilde{N}_{\theta}\left(r_{T E 1} r_{T E 2} e^{-i k_{z} z}+r_{T E 2} e^{i k_{z} z}\right)+\mathbf{e}_{z} \widetilde{N}_{z}\left(r_{T M 1} r_{T M 2} e^{-i k_{z} z}+r_{T M 2} e^{i k_{z} z}\right)\right],
\end{aligned}
$$

where

$$
\begin{aligned}
& \widetilde{N_{r}}=\frac{i}{8 \pi^{2}}\left(k_{r} d_{z}+k_{z} d_{r}\right), \\
& \widetilde{N_{\theta}}=\frac{i}{8 \pi^{2}} \frac{\omega^{2}}{k_{z}} d_{\theta}, \\
& \widetilde{N_{z}}=\frac{k_{r}}{k_{z}} \widetilde{N_{r}} .
\end{aligned}
$$


The electric field $\mathbf{E}(\mathbf{r})$ from a dipole source at the point $\left(0,0, z_{0}\right)$ between two dielectric half-spaces separated by a distance $d$ is the sum of (83) and (84):

$$
\mathbf{E}(\mathbf{r})=\mathbf{E}_{u p}(\mathbf{r})+\mathbf{E}_{\text {down }}(\mathbf{r}) .
$$

It is convenient to write local components of electric Green functions for a given $\mathbf{k}_{\|}$in a local basis $\mathbf{e}_{r}, \mathbf{e}_{\theta}, \mathbf{e}_{z}$ :

$$
\begin{gathered}
D_{r r}^{E}\left(\omega, k_{r}\right)=\frac{i k_{z}}{2 \Delta_{T M}}\left[e^{i k_{z} z}\left(r_{T M 1} r_{T M 2} e^{i k_{z}\left(2 d-z_{0}\right)}-r_{T M 2} e^{i k_{z} z_{0}}\right)+\right. \\
\left.e^{-i k_{z} z}\left(r_{T M 1} r_{T M 2} e^{i k_{z}\left(2 d+z_{0}\right)}-r_{T M 1} e^{i k_{z}\left(2 d-z_{0}\right)}\right)\right], \\
D_{\theta \theta}^{E}\left(\omega, k_{r}\right)=\frac{i \omega^{2}}{2 k_{z} \Delta_{T E}}\left[e^{i k_{z} z}\left(r_{T E 1} r_{T E 2} e^{i k_{z}\left(2 d-z_{0}\right)}+r_{T E 2} e^{i k_{z} z_{0}}\right)+\right. \\
\left.e^{-i k_{z} z}\left(r_{T E 1} r_{T E 2} e^{i k_{z}\left(2 d+z_{0}\right)}+r_{T E 1} e^{i k_{z}\left(2 d-z_{0}\right)}\right)\right], \\
D_{z z}^{E}\left(\omega, k_{r}\right)=\frac{i k_{r}^{2}}{2 k_{z} \Delta_{T M}}\left[e ^ { i k _ { z } z } \left(r_{T M 1} r_{T M 2} e^{i k_{z}\left(2 d-z_{0}\right)}+r_{T M 2} e^{\left.i k_{z} z_{0}\right)}+\right.\right. \\
\left.e^{-i k_{z} z}\left(r_{T M 1} r_{T M 2} e^{i k_{z}\left(2 d+z_{0}\right)}+r_{T M 1} e^{i k_{z}\left(2 d-z_{0}\right)}\right)\right],
\end{gathered}
$$

where we defined $\Delta_{T M} \equiv 1-r_{T M 1} r_{T M 2} e^{2 i k_{z} d}, \Delta_{T E} \equiv 1-r_{T E 1} r_{T E 2} e^{2 i k_{z} d}$. One derives components of electric Green functions from (89)-(91):

$$
\begin{aligned}
& D_{x x}^{E}\left(\omega, \mathbf{r}, \mathbf{r}^{\prime}\right)=\int\left(D_{r r}^{E}\left(\omega, k_{r}\right) \cos ^{2} \theta+D_{\theta \theta}^{E}\left(\omega, k_{r}\right) \sin ^{2} \theta\right) e^{i \mathbf{k}_{\|}\left(\mathbf{r}_{\|}-\mathbf{r}_{\|}^{\prime}\right)} \frac{d^{2} \mathbf{k}_{\|}}{(2 \pi)^{2}}, \\
& D_{y y}^{E}\left(\omega, \mathbf{r}, \mathbf{r}^{\prime}\right)=\int\left(D_{r r}^{E}\left(\omega, k_{r}\right) \sin ^{2} \theta+D_{\theta \theta}^{E}\left(\omega, k_{r}\right) \cos ^{2} \theta\right) e^{i \mathbf{k}_{\|}\left(\mathbf{r}_{\|}-\mathbf{r}_{\|}^{\prime}\right)} \frac{d^{2} \mathbf{k}_{\|}}{(2 \pi)^{2}}, \\
& D_{z z}^{E}\left(\omega, \mathbf{r}, \mathbf{r}^{\prime}\right)=\int D_{z z}^{E}\left(\omega, k_{r}\right) e^{i \mathbf{k}_{\|}\left(\mathbf{r}_{\|}-\mathbf{r}_{\|}^{\prime}\right)} \frac{d^{2} \mathbf{k}_{\|}}{(2 \pi)^{2}} .
\end{aligned}
$$

Electric Green functions (92)-(94) agree with electric Green functions from Ref. [63]. Magnetic Green function can be evaluated from electric Green function:

$$
D_{i l}^{H}\left(\omega, \mathbf{x}, \mathbf{x}^{\prime}\right)=\frac{1}{\omega^{2}} \epsilon_{i j k} \epsilon_{l m n} \frac{\partial}{\partial x^{j}} \frac{\partial}{\partial x^{\prime m}} D_{k n}^{E}\left(\omega, \mathbf{x}, \mathbf{x}^{\prime}\right) .
$$

One can find local components of magnetic Green functions for a given $\mathbf{k}_{\|}$in a local basis $\mathbf{e}_{r}, \mathbf{e}_{\theta}, \mathbf{e}_{z}$ :

$$
\begin{gathered}
D_{r r}^{H}\left(\omega, k_{r}\right)=\frac{i k_{z}}{2 \Delta_{T E}}\left[e^{i k_{z} z}\left(r_{T E 1} r_{T E 2} e^{i k_{z}\left(2 d-z_{0}\right)}-r_{T E 2} e^{i k_{z} z_{0}}\right)+\right. \\
\left.e^{-i k_{z} z}\left(r_{T E 1} r_{T E 2} e^{i k_{z}\left(2 d+z_{0}\right)}-r_{T E 1} e^{i k_{z}\left(2 d-z_{0}\right)}\right)\right], \\
D_{\theta \theta}^{H}\left(\omega, k_{r}\right)=\frac{i \omega^{2}}{2 k_{z} \Delta_{T M}}\left[e^{i k_{z} z}\left(r_{T M 1} r_{T M 2} e^{i k_{z}\left(2 d-z_{0}\right)}+r_{T M 2} e^{i k_{z} z_{0}}\right)+\right. \\
\left.e^{-i k_{z} z}\left(r_{T M 1} r_{T M 2} e^{i k_{z}\left(2 d+z_{0}\right)}+r_{T M 1} e^{i k_{z}\left(2 d-z_{0}\right)}\right)\right], \\
D_{z z}^{H}\left(\omega, k_{r}\right)=\frac{i k_{r}^{2}}{2 k_{z} \Delta_{T E}}\left[e^{i k_{z} z}\left(r_{T E 1} r_{T E 2} e^{i k_{z}\left(2 d-z_{0}\right)}+r_{T E 2} e^{i k_{z} z_{0}}\right)+\right. \\
\left.e^{-i k_{z} z}\left(r_{T E 1} r_{T E 2} e^{i k_{z}\left(2 d+z_{0}\right)}+r_{T E 1} e^{i k_{z}\left(2 d-z_{0}\right)}\right)\right] .
\end{gathered}
$$


The components $D_{i i}^{H}\left(\omega, \mathbf{r}, \mathbf{r}^{\prime}\right)$ can be evaluated from local components (96)-(98) in complete analogy to (92)-(94). One can check that for every $0<z_{0}<d$

$$
\begin{aligned}
& D_{\theta \theta}^{E}\left(z_{0}, z_{0}\right)+D_{r r}^{H}\left(z_{0}, z_{0}\right)-D_{z z}^{H}\left(z_{0}, z_{0}\right)=\frac{2 i k_{z} r_{T E 1} r_{T E 2} e^{2 i k_{z} d}}{\Delta_{T E}}, \\
& D_{r r}^{E}\left(z_{0}, z_{0}\right)-D_{z z}^{E}\left(z_{0}, z_{0}\right)+D_{\theta \theta}^{H}\left(z_{0}, z_{0}\right)=\frac{2 i k_{z} r_{T M 1} r_{T M 2} e^{2 i k_{z} d}}{\Delta_{T M}} .
\end{aligned}
$$

$T_{z z}$ component of the energy-momentum tensor is expressed in terms of electric and magnetic Green functions:

$$
\begin{array}{r}
T_{z z}\left(z_{0}\right)=-\frac{i}{2} \int_{-\infty}^{+\infty} \frac{d \omega}{2 \pi}\left[D_{x x}^{E}\left(z_{0}, z_{0}\right)+D_{y y}^{E}\left(z_{0}, z_{0}\right)-D_{z z}^{E}\left(z_{0}, z_{0}\right)+\right. \\
\left.D_{x x}^{H}\left(z_{0}, z_{0}\right)+D_{y y}^{H}\left(z_{0}, z_{0}\right)-D_{z z}^{H}\left(z_{0}, z_{0}\right)\right]
\end{array}
$$

We use identities (99), (100) to derive the Casimir pressure at zero temperature between two dielectric half-spaces separated by a distance $d$ :

$$
\begin{aligned}
P=T_{z z}\left(z_{0}\right)=-\frac{1}{2 \pi^{2}} \int_{0}^{\infty} & d \omega \int_{0}^{\infty} d k_{r} k_{r} \exp \left(-2 \sqrt{\omega^{2}+k_{r}^{2}} d\right) \sqrt{\omega^{2}+k_{r}^{2}} \times \\
& \left(\frac{r_{T E 1}\left(i \omega, k_{r}\right) r_{T E 2}\left(i \omega, k_{r}\right)}{\Delta_{T E}\left(i \omega, k_{r}\right)}+\frac{r_{T M 1}\left(i \omega, k_{r}\right) r_{T M 2}\left(i \omega, k_{r}\right)}{\Delta_{T M}\left(i \omega, k_{r}\right)}\right) .
\end{aligned}
$$

Casimir pressure between two dielectric half-spaces separated by a vacuum slit (102) was derived in Ref. [3] by use of Green functions and fluctuation-dissipation theorem. The Casimir effect theory based on Green functions was further developed in Refs. [4,64-69].

In Ref. [4] the Lifshitz force is expressed through $T_{z z}$ component of the energymomentum tensor and, by making use of fluctuation-dissipation theorem, through retarded Green functions. Furthermore, in Ref. [4] authors search solution for Green functions between two dielectric half-spaces which does not depend on $z+z_{0}$, where $z$ and $z_{0}$ are two arguments of the Green function in real space. In our opinion, an important physical part of the solution is therefore not present in Ref. [4]. Although one obtains the result for standard Lifshitz pressure (102) in Ref. [4], absence of $z+z_{0}$ terms in Green functions in Ref. [4] does not give correct values of propagators at coincident points $z=z_{0}$ to derive the Casimir-Polder potential of a neutral atom between two dielectric half-spaces. In our derivation performed in this section $z+z_{0}$ terms are rigorously derived for all Green functions. In Section 4 we derive expression for the Casimir-Polder potential of a neutral atom between two dielectric half-spaces with parallel boundaries.

\section{Casimir-Polder Potential of Anisotropic Atom between Two Dielectric Half-Spaces}

We use expression (46) and electric propagators for two half-spaces (92)-(94) at $\mathbf{r}_{\|}=\mathbf{r}_{\| \prime}^{\prime}$, $z=z_{0} \equiv L$ to derive the Casimir-Polder potential of a neutral anisotropic atom between two dielectric half-spaces with parallel boundaries separated by a distance $d$ :

$$
\begin{gathered}
U(L, d)=-\int_{0}^{\infty} \frac{d \omega}{2 \pi} \int_{0}^{\infty} \frac{d k_{r} k_{r}}{2 \pi}\left[\frac { \operatorname { e x p } ( - 2 \sqrt { \omega ^ { 2 } + k _ { r } ^ { 2 } } L ) } { 2 \sqrt { \omega ^ { 2 } + k _ { r } ^ { 2 } } } \left[\frac{r_{T M 2}\left(i \omega, k_{r}\right)}{\Delta_{T M}\left(i \omega, k_{r}\right)} k_{r}^{2} \alpha_{z z}(i \omega)+\right.\right. \\
\left.\frac{1}{2}\left(\frac{r_{T M 2}\left(i \omega, k_{r}\right)}{\Delta_{T M}\left(i \omega, k_{r}\right)}\left(\omega^{2}+k_{r}^{2}\right)-\frac{r_{T E 2}\left(i \omega, k_{r}\right)}{\Delta_{T E}\left(i \omega, k_{r}\right)} \omega^{2}\right)\left(\alpha_{x x}(i \omega)+\alpha_{y y}(i \omega)\right)\right]+ \\
\frac{\exp \left(-2 \sqrt{\omega^{2}+k_{r}^{2}}(d-L)\right)}{2 \sqrt{\omega^{2}+k_{r}^{2}}}\left[\frac{r_{T M 1}\left(i \omega, k_{r}\right)}{\Delta_{T M}\left(i \omega, k_{r}\right)} k_{r}^{2} \alpha_{z z}(i \omega)+\right. \\
\left.\left.\frac{1}{2}\left(\frac{r_{T M 1}\left(i \omega, k_{r}\right)}{\Delta_{T M}\left(i \omega, k_{r}\right)}\left(\omega^{2}+k_{r}^{2}\right)-\frac{r_{T E 1}\left(i \omega, k_{r}\right)}{\Delta_{T E}\left(i \omega, k_{r}\right)} \omega^{2}\right)\left(\alpha_{x x}(i \omega)+\alpha_{y y}(i \omega)\right)\right]\right]+U_{2}(d),
\end{gathered}
$$


where

$$
\begin{aligned}
U_{2}(d)= & \int_{0}^{\infty} \frac{d \omega}{2 \pi} \int_{0}^{\infty} \frac{d k_{r} k_{r}}{2 \pi} \frac{\exp \left(-2 \sqrt{\omega^{2}+k_{r}^{2}} d\right)}{2 \sqrt{\omega^{2}+k_{r}^{2}}}\left[\left(\alpha_{x x}(i \omega)+\alpha_{y y}(i \omega)\right) \times\right. \\
& \left(\frac{r_{T M 1}\left(i \omega, k_{r}\right) r_{T M 2}\left(i \omega, k_{r}\right)}{\Delta_{T M}\left(i \omega, k_{r}\right)}\left(\omega^{2}+k_{r}^{2}\right)+\frac{r_{T E 1}\left(i \omega, k_{r}\right) r_{T E 2}\left(i \omega, k_{r}\right)}{\Delta_{T E}\left(i \omega, k_{r}\right)} \omega^{2}\right) \\
& \left.-2 \frac{r_{T M 1}\left(i \omega, k_{r}\right) r_{T M 2}\left(i \omega, k_{r}\right)}{\Delta_{T M}\left(i \omega, k_{r}\right)} k_{r}^{2} \alpha_{z z}(i \omega)\right] .
\end{aligned}
$$

Quantum-electrodynamic level shifts of anisotropic atom between parallel perfectly conducting mirrors are considered in Ref. [70]. To proceed it is convenient to introduce a dimensionless variable $\xi=\pi(2 L-d) / d$. We substitute reflection coefficients $r_{T M}=1$, $r_{T E}=-1$ to (103) and use integrals

$$
\begin{aligned}
& \int_{0}^{\infty} \frac{d \rho \rho^{3}}{\exp (2 \pi \rho)-1}=\frac{1}{240}, \\
& \int_{0}^{\infty} d \rho \rho^{3} \frac{\cosh \xi \rho}{\sinh \pi \rho}=\frac{3-2 \cos ^{2}(\xi / 2)}{8 \cos ^{4}(\xi / 2)}
\end{aligned}
$$

to obtain the result which agrees with Barton [70] for the ground state energy of anisotropic atom at large distances from perfectly conducting mirrors:

$$
\begin{aligned}
U(\xi, d)= & -\frac{\pi^{2}}{12 d^{4}}\left(\alpha_{x x}(0)+\alpha_{y y}(0)+\alpha_{z z}(0)\right) \frac{3-2 \cos ^{2}(\xi / 2)}{8 \cos ^{4}(\xi / 2)}+ \\
& \frac{\pi^{2}}{1440 d^{4}}\left(\alpha_{x x}(0)+\alpha_{y y}(0)-\alpha_{z z}(0)\right) .
\end{aligned}
$$

The result (107) is valid at large distances of the atom from mirrors $L, d-L \gg \lambda_{0} \equiv 2 \pi / \omega_{0}$, $\lambda_{0}$ is a wavelength corresponding to a typical absorption frequency $\omega_{0}$. At large distances from materials one can effectively take atomic polarizabilities at zero frequencies: the result (107) depends on $\alpha_{x x}(0), \alpha_{y y}(0), \alpha_{z z}(0)$.

Various confined cases have been studied before: Casimir interaction of two rectangular and circular cylinders between two parallel plates [71,72], the Casimir-Polder effect for a perfectly conducting wedge [49], interaction of compact objects with a mirror plane due to quantum fluctuations of a scalar or electromagnetic field [73], Casimir-Polder interaction of two anisotropic atoms in front of a perfectly conducting plate as well as Casimir interaction of two perfect spheres in front of a perfectly conducting plate [74], Casimir interactions of an object inside a spherical metal shell [75], Casimir interaction of objects inside a perfectly conducting cylindrical shell [76].

\section{Conducting $2+1$ Layer}

The conducting plane $2+1$ layer with surface conductivity $\sigma(\omega)$ at the boundary of a dielectric half-space can be introduced by imposing boundary conditions

$$
\begin{aligned}
\left(\mathbf{E}^{m}-\mathbf{E}^{v}\right) \times \mathbf{n} & =0, \\
\left(\mathbf{H}^{m}-\mathbf{H}^{v}\right) \times \mathbf{n} & =\sigma(\omega) \mathbf{E}^{v} .
\end{aligned}
$$

Here $\mathbf{E}^{m}, \mathbf{H}^{m}$ and $\mathbf{E}^{v}, \mathbf{H}^{v}$ are electric and magnetic fields in the medium and vacuum, respectively. We consider diffraction from a layer $z=d$ at the boundary of a dielectric halfspace $z>d, \mathbf{n}=(0,0,-1)$. We work in the same system of coordinates as in the previous section and use the same notations. From boundary conditions (108), (109), transversality 
of fields and expansions (51)-(56) one writes system of equations for a given $\mathbf{k}_{\|}$in a local basis $\mathbf{e}_{r}, \mathbf{e}_{\theta}, \mathbf{e}_{z}$ :

$$
\begin{aligned}
& u_{1 r} k_{r}+K_{z 1} u_{1 z}=0, \\
& v_{1 r} k_{r}-k_{z} v_{1 z}=0, \\
& v_{1 r} e^{-i k_{z} d}+N_{r} e^{i k_{z}\left(d-z_{0}\right)}=u_{1 r} e^{i K_{z 1} d}, \\
& v_{1 \theta} e^{-i k_{z} d}+N_{\theta} e^{i k_{z}\left(d-z_{0}\right)}=u_{1 \theta} e^{i K_{z 1} d}, \\
&-K_{z 1} u_{1 \theta} e^{i K_{z 1} d}=k_{z} v_{1 \theta} e^{-i k_{z} d}+M_{r} e^{i k_{z}\left(d-z_{0}\right)}+\sigma(\omega) \omega\left(v_{1 \theta} e^{-i k_{z} d}+N_{\theta} e^{i k_{z}\left(d-z_{0}\right)}\right), \\
& \sigma(\omega) \omega\left(v_{1 r} e^{-i k_{z} d}+N_{r} e^{i k_{z}\left(d-z_{0}\right)}\right)-k_{r} u_{1 z} e^{i K_{z 1} d}+K_{z 1} u_{1 r} e^{i K_{z 1} d}= \\
&-k_{r} v_{1 z} e^{-i k_{z} d}-k_{z} v_{1 r} e^{-i k_{z} d}+M_{\theta} e^{i k_{z}\left(d-z_{0}\right)} .
\end{aligned}
$$

One finds

$$
\begin{aligned}
& v_{1 r}=-N_{r} r_{T M 1} e^{i k_{z}\left(2 d-z_{0}\right)}, \\
& v_{1 \theta}=N_{\theta} r_{T E 1} e^{i k_{z}\left(2 d-z_{0}\right)}, \\
& v_{1 z}=N_{z} r_{T M 1} e^{i k_{z}\left(2 d-z_{0}\right)},
\end{aligned}
$$

where

$$
\begin{aligned}
r_{T M 1} & =\frac{\sigma(\omega) \omega k_{z} K_{z 1}+\omega^{2}\left(\varepsilon k_{z}-K_{z 1}\right)}{\sigma(\omega) \omega k_{z} K_{z 1}+\omega^{2}\left(\varepsilon k_{z}+K_{z 1}\right)}, \\
r_{T E 1} & =\frac{\sigma(\omega) \omega+k_{z}-K_{z 1}}{\sigma(\omega) \omega+k_{z}+K_{z 1}} .
\end{aligned}
$$

One can find reflection of electromagnetic field from a conducting layer located at $z=0$ in a similar way. Then it is immediate to see that Casimir and Casimir-Polder formulas derived in previous sections are valid in the present case if one substitutes reflection coefficients (119) and (120). Conducting planes with constant conductivities have been studied in Refs. [77,78].

Non-diagonal components of the surface conductivity are also important for applications. The Casimir force between $2+1$ layers in the quantum Hall regime described by quantized conductivities of the layers has been studied in Ref. [79], the force is repulsive for layers with the same type of carrier. Chern insulator is an example of $2+1$ layer with a conductivity quantized when no external magnetic field is present [80]. Please note that the Casimir force between identical Chern insulator plates with vanishing longitudinal conductivities and small values of non-diagonal conductivities is repulsive; however, for large values of non-diagonal conductivities the Casimir force is attractive and coincides with the Casimir force between two perfectly conducting plates in the limit of infinite conductivities [42]. In Ref. [43] general result for the Casimir energy of two plates with arbitrary values of non-diagonal constant conductivities is derived. A review of Casimir physics for novel materials including Chern and topological insulators can be found in Ref. [17]. 


\section{Polarization Operator of $2+1$ Fermions and Graphene}

Spatial dispersion yields possibility to generalize boundary condition (109). For systems invariant under rotation around $z$ axis one can introduce longitudinal and transverse conductivities $\sigma_{r}\left(\omega, k_{r}\right), \sigma_{\theta}\left(\omega, k_{r}\right)$ and write boundary conditions:

$$
\begin{aligned}
\left(\mathbf{E}^{m}-\mathbf{E}^{v}\right) \times \mathbf{n} & =0 \\
{\left[\left(\mathbf{H}^{m}\left(\omega, \mathbf{k}_{\|}\right)-\mathbf{H}^{v}\left(\omega, \mathbf{k}_{\|}\right)\right) \times \mathbf{n}\right]_{r} } & =\sigma_{r}\left(\omega, k_{r}\right) E_{r}^{v}\left(\omega, \mathbf{k}_{\|}\right) \\
{\left[\left(\mathbf{H}^{m}\left(\omega, \mathbf{k}_{\|}\right)-\mathbf{H}^{v}\left(\omega, \mathbf{k}_{\|}\right)\right) \times \mathbf{n}\right]_{\theta} } & =\sigma_{\theta}\left(\omega, k_{r}\right) E_{\theta}^{v}\left(\omega, \mathbf{k}_{\|}\right)
\end{aligned}
$$

Boundary conditions (122), (123) yield equations

$$
\begin{aligned}
& -K_{z 1} u_{1 \theta} e^{i K_{z 1} d}=k_{z} v_{1 \theta} e^{-i k_{z} d}+M_{r} e^{i k_{z}\left(d-z_{0}\right)}+\sigma_{\theta}\left(\omega, k_{r}\right) \omega\left(v_{1 \theta} e^{-i k_{z} d}+N_{\theta} e^{i k_{z}\left(d-z_{0}\right)}\right), \\
& \sigma_{r}\left(\omega, k_{r}\right) \omega\left(v_{1 r} e^{-i k_{z} d}+N_{r} e^{i k_{z}\left(d-z_{0}\right)}\right)-k_{r} u_{1 z} e^{i K_{z 1} d}+K_{z 1} u_{1 r} e^{i K_{z 1} d}= \\
& -k_{r} v_{1 z} e^{-i k_{z} d}-k_{z} v_{1 r} e^{-i k_{z} d}+M_{\theta} e^{i k_{z}\left(d-z_{0}\right)} \text {. }
\end{aligned}
$$

The solution of Equations (110)-(113), (124) and (125) can be written in the form

$$
\begin{aligned}
& v_{1 r}=-N_{r} r_{T M 1} e^{i k_{z}\left(2 d-z_{0}\right),} \\
& v_{1 \theta}=N_{\theta} r_{T E 1} e^{i k_{z}\left(2 d-z_{0}\right),} \\
& v_{1 z}=N_{z} r_{T M 1} e^{i k_{z}\left(2 d-z_{0}\right),}
\end{aligned}
$$

where now reflection coefficients $r_{T M 1}, r_{T E 1}$ depend on two different conductivities $\sigma_{r}\left(\omega, k_{r}\right)$ and $\sigma_{\theta}\left(\omega, k_{r}\right)$ :

$$
\begin{aligned}
r_{T M 1} & =\frac{\sigma_{r}\left(\omega, k_{r}\right) \omega k_{z} K_{z 1}+\omega^{2}\left(\varepsilon k_{z}-K_{z 1}\right)}{\sigma_{r}\left(\omega, k_{r}\right) \omega k_{z} K_{z 1}+\omega^{2}\left(\varepsilon k_{z}+K_{z 1}\right)}, \\
r_{T E 1} & =\frac{\sigma_{\theta}\left(\omega, k_{r}\right) \omega+k_{z}-K_{z 1}}{\sigma_{\theta}\left(\omega, k_{r}\right) \omega+k_{z}+K_{z 1}} .
\end{aligned}
$$

Please note it is impossible to define two conductivities with a frequency dispersion for a given layer. One needs vector $\mathbf{k}_{\|}$to determine spatial direction of the incident wave and define longitudinal and transverse conductivities.

As soon as one determines conductivities $\sigma_{r}\left(\omega, k_{r}\right), \sigma_{\theta}\left(\omega, k_{r}\right)$ one can find the Casimir force and Casimir-Polder potentials by direct substitution of the coefficients (129), (130) to general results (50), (102) and (103).

Now we express conductivities $\sigma_{r}\left(\omega, k_{r}\right), \sigma_{\theta}\left(\omega, k_{r}\right)$ in terms of components of polarization operator for $2+1$-dimensional fermions. Consider polarization operator $\Pi^{\nu \rho}\left(\omega, \mathbf{k}_{\|}\right)$ of $2+1$-dimensional fermions in the plane layer $z=0$. One can evaluate the components of the polarization operator in the plane layer and then extend it to $3+1$ space by condition $\Pi^{\mu 3}=\Pi^{3 \mu}=0$.

We first find reflection coefficients from the plane layer $z=0$ of $2+1$-dimensional fermions in vacuum without specifying a concrete form of the polarization operator for $2+1$-dimensional fermions in a layer. One can write equations for electromagnetic field in this case:

$$
\partial_{\mu} F^{\mu v}+\delta(z) \Pi^{v \rho} A_{\rho}=0,
$$

where $F^{\mu v}=\partial^{\mu} A^{v}-\partial^{v} A^{\mu}$. Equations (131) lead to conditions

$$
\left.\partial_{z} A_{m}\right|_{z=+0}-\left.\partial_{z} A_{m}\right|_{z=-0}=\left.\Pi_{m n} A^{n}\right|_{z=0} .
$$


Gauge potentials should be continuous through $z=0$ :

$$
\left.A_{m}\right|_{z=+0}=\left.A_{m}\right|_{z=-0} .
$$

We select $A_{z}=0$ gauge for convenience. Then equations for reflection of the TE wave may be written as follows:

$$
\begin{aligned}
& A_{\theta}=e^{i k_{r} r} e^{i k_{z} z}+r_{T E} e^{i k_{r} r} e^{-i k_{z} z} \text { for } z<0, \\
& A_{\theta}=e^{i k_{z} z} e^{i k_{r} r} t_{T E} \text { for } z>0,
\end{aligned}
$$

and one obtains

$$
\Pi_{\theta n} A^{n}=-\Pi_{\theta \theta}\left(\omega, k_{r}\right) A_{\theta} .
$$

Continuity of vector potentials at $z=0$ yields $1+r_{T E}=t_{T E}$. From the condition (132) one derives

$$
r_{T E}\left(\omega, k_{r}\right)=\frac{\Pi_{\theta \theta}\left(\omega, k_{r}\right)}{-2 i k_{z}-\Pi_{\theta \theta}\left(\omega, k_{r}\right)} .
$$

Please note that conditions $A_{\theta}=0, \omega A_{0}=k_{r} A_{r}$ are imposed on TM wave. For $A_{0}$ one can write

$$
\begin{aligned}
& A_{0}=e^{i k_{r} r} e^{i k_{z} z}+r_{A_{0}} e^{i k_{r} r} e^{-i k_{z} z} \text { for } z<0 \\
& A_{0}=e^{i k_{z} z} e^{i k_{r} r} t_{A_{0}} \text { for } z>0 .
\end{aligned}
$$

Current conservation yields conditions

$$
\begin{aligned}
\omega \Pi_{00}\left(\omega, k_{r}\right)-k_{r} \Pi_{r 0}\left(\omega, k_{r}\right) & =0, \\
\omega \Pi_{0 r}\left(\omega, k_{r}\right)-k_{r} \Pi_{r r}\left(\omega, k_{r}\right) & =0,
\end{aligned}
$$

which result in

$$
\omega^{2} \Pi_{00}\left(\omega, k_{r}\right)=k_{r}^{2} \Pi_{r r}\left(\omega, k_{r}\right) .
$$

Now we can write

$$
\Pi_{00} A^{0}+\Pi_{0 r} A^{r}=-\frac{k_{z}^{2}}{\omega^{2}} \Pi_{r r} A_{0}=-\frac{k_{z}^{2}}{\omega^{2}} \Pi_{r r} A_{0} .
$$

Equation (132) results in

$$
2 i k_{z}\left(t_{A_{0}}+r_{A_{0}}-1\right)=-\frac{k_{z}^{2}}{\omega^{2}} \Pi_{r r} A_{0}\left(1+r_{A_{0}}\right) .
$$

From equality $r_{T M}\left(\omega, k_{r}\right)=-r_{A_{0}}\left(\omega, k_{r}\right)$ one obtains

$$
r_{T M}\left(\omega, k_{r}\right)=\frac{i k_{z} \Pi_{r r}\left(\omega, k_{r}\right)}{-2 \omega^{2}+i k_{z} \Pi_{r r}\left(\omega, k_{r}\right)} .
$$

By making use of identities

$$
\begin{aligned}
& \sigma_{r}\left(\omega, k_{r}\right)=\frac{-i \Pi_{r r}\left(\omega, k_{r}\right)}{\omega}, \\
& \sigma_{\theta}\left(\omega, k_{r}\right)=\frac{-i \Pi_{\theta \theta}\left(\omega, k_{r}\right)}{\omega}
\end{aligned}
$$

one can check that reflection coefficients (137), (143) coincide with reflection coefficients (129), (130) for $\varepsilon=1$.

Reflection coefficients of electromagnetic field from a plane layer of $2+1$-dimensional fermions at the boundary of a dielectric half-space with a frequency dispersion of per- 
mittivity $\varepsilon \equiv \varepsilon(\omega)$ can be immediately expressed in terms of components of polarization operator $\Pi_{v \rho}\left(\omega, k_{r}\right)$ for $2+1$-dimensional fermions in a layer:

$$
\begin{aligned}
r_{T M} & =\frac{-i \Pi_{r r}\left(\omega, k_{r}\right) k_{z} K_{z}+\omega^{2}\left(\varepsilon k_{z}-K_{z}\right)}{-i \Pi_{r r}\left(\omega, k_{r}\right) k_{z} K_{z}+\omega^{2}\left(\varepsilon k_{z}+K_{z}\right)}, \\
r_{T E} & =\frac{-i \Pi_{\theta \theta}\left(\omega, k_{r}\right)+k_{z}-K_{z}}{-i \prod_{\theta \theta}\left(\omega, k_{r}\right)+k_{z}+K_{z}} .
\end{aligned}
$$

The Casimir force and the Casimir-Polder potential of an anisotropic atom can be found by substitution of reflection coefficients (146), (147) to expressions (50), (102) and (103).

We define $\operatorname{tr} \Pi \equiv \Pi_{m}^{m}$. By making use of Equation (140) and equality

$$
\operatorname{tr} \Pi\left(\omega, k_{r}\right)=\Pi_{00}\left(\omega, k_{r}\right) \frac{k_{r}^{2}-\omega^{2}}{k_{r}^{2}}-\Pi_{\theta \theta}\left(\omega, k_{r}\right)
$$

one can express reflection coefficients (146) and (147) in terms of $\Pi_{00}\left(\omega, k_{r}\right)$ and $\operatorname{tr} \Pi\left(\omega, k_{r}\right)$.

In the case of a fermion layer in a vacuum $(\varepsilon=1)$ we obtain reflection coefficients in a form first given in [81]:

$$
r_{T M}\left(\omega, k_{r}\right)=\frac{k_{z} \Pi_{00}}{k_{z} \Pi_{00}+2 i k_{r}^{2}}, \quad r_{T E}\left(\omega, k_{r}\right)=-\frac{k_{z}^{2} \Pi_{00}+k_{r}^{2} \operatorname{tr} \Pi}{k_{z}^{2} \Pi_{00}+k_{r}^{2}\left(\operatorname{tr} \Pi-2 i k_{z}\right)} .
$$

Components of polarization operator for $2+1$ Dirac fermions at finite temperature were first evaluated in Ref. [81] during evaluation of the Casimir free energy of a graphene layer-metal half-space system.

Graphene is a system of $2+1$ Dirac fermions with a linear dispersion law $\omega=$ $v_{F} k\left(v_{F} \approx c / 300\right.$ is a Fermi velocity, $c$ is a speed of light). This description is valid for quasiparticles of pristine graphene $[82,83]$ at energies less than $2 \mathrm{eV}$. There are $N=4$ species of fermions in graphene.

Interaction of ideal metal with graphene layer at temperature $T=0$ is studied in Ref. [84]. Finite temperature Casimir interaction in graphene layer-metal systems including ideal metal and Au half-space is studied in Ref. [81]. One can write finite temperature expansions for $k_{r} \rightarrow 0$ (for zero mass gap and zero chemical potential):

$$
\begin{aligned}
& \Pi^{00}\left(i \omega=0, k_{r}\right)=\frac{4 \alpha N T \ln 2}{v_{F}^{2}}+\frac{\alpha N k_{r}^{2}}{12 T}+\ldots, \\
& \operatorname{tr} \Pi\left(i \omega=0, k_{r}\right)-\Pi^{00}\left(i \omega=0, k_{r}\right)=\frac{\alpha N v_{F}^{2} k_{r}^{2}}{6 T}+\ldots,
\end{aligned}
$$

$\alpha$ is the coupling constant, which lead to the following high-temperature behavior of the free energy in the graphene-ideal metal system (higher order terms in $v_{F}$ are omitted):

$$
\begin{aligned}
\mathcal{F}_{0 T M} & =-\frac{T \zeta(3)}{16 \pi a^{2}}+\ldots \\
\mathcal{F}_{0 T E} & =-\frac{\alpha N v_{F}^{2}}{192 \pi a^{3}}+\ldots
\end{aligned}
$$

Please note that

$$
\left.\mathcal{F}_{\text {Drude }}\right|_{T \rightarrow \infty}=\left.\frac{1}{2} \mathcal{F}_{\text {id }}\right|_{T \rightarrow \infty}=-\frac{T \zeta(3)}{16 \pi a^{2}}
$$

is the high-temperature metal-metal asymptotics of the free energy for a Drude model of permittivity, which is also one half of the high-temperature asymptotics of the free energy for perfectly conducting boundary conditions. Detailed analysis [81] has demonstrated that the high-temperature behavior in the graphene layer-metal system can be observed at separations of the order $100 \mathrm{~nm}$ at room temperature. 
In Ref. [85] the Casimir force in the system of an Au-coated sphere and a graphene layer deposited on a $\mathrm{SiO}_{2}$ film covering a $\mathrm{Si}$ plate is measured. Experimental results of Ref. [85] are found to be in a good agreement with the theory based on the polarization operator of graphene.

Planar QED at finite temperature and density and its relation to minimal conductivity of graphene is considered in Ref. [86]. The origin of the high-temperature behavior (152) for 2D materials with a frequency dispersion is discussed in Ref. [87]. In Ref. [88] a convenient representation of graphene polarization operator is applied at nonzero temperature to study properties of graphene reflectivity at real frequencies. A review of recent results in graphene physics obtained by use of polarization operator can be found in Ref. [89].

\section{Impedance Boundary Conditions}

Impedance boundary conditions are defined as follows:

$$
\mathbf{E}_{t}=\zeta(\omega)\left[\mathbf{H}_{t} \times \mathbf{n}\right] .
$$

Tangential components of the electromagnetic field are continuous through the boundary of metal in this case. For impedance boundary conditions (155) one must write three equations to determine reflected electromagnetic field. We impose impedance boundary conditions at $z=d$ and consider reflection of an electromagnetic field (51), (52) from a metallic half-space $z \geq d$. The reflected electric field is transverse:

$$
v_{1 r} k_{r}-v_{1 z} k_{z}=0 .
$$

Impedance boundary conditions (155) and expansions (51)-(54) yield two equations in a local basis $\mathbf{e}_{r}, \mathbf{e}_{\theta}, \mathbf{e}_{z}$ :

$$
\begin{aligned}
& N_{r} e^{i k_{z}\left(d-z_{0}\right)}+v_{1 r} e^{-i k_{z} d}=-\zeta(\omega)\left(-k_{r} v_{1 z} e^{-i k_{z} d}-k_{z} v_{1 r}+M_{\theta} e^{i k_{z}\left(d-z_{0}\right)}\right), \\
& N_{\theta} e^{i k_{z}\left(d-z_{0}\right)}+v_{1 \theta} e^{-i k_{z} d}=\zeta(\omega)\left(k_{z} v_{1 \theta} e^{-i k_{z} d}+M_{r} e^{i k_{z}\left(d-z_{0}\right)}\right) .
\end{aligned}
$$

One finds in this case

$$
\begin{aligned}
& v_{1 r}=-N_{r} r_{T M 1} e^{i k_{z}\left(2 d-z_{0}\right)}, \\
& v_{1 \theta}=N_{\theta} r_{T E 1} e^{i k_{z}\left(2 d-z_{0}\right)}, \\
& v_{1 z}=N_{z} r_{T M 1} e^{i k_{z}\left(2 d-z_{0}\right)},
\end{aligned}
$$

where

$$
\begin{aligned}
r_{T M 1} & =\frac{k_{z}-\zeta(\omega) \omega}{k_{z}+\zeta(\omega) \omega}, \\
r_{T E 1} & =\frac{\zeta(\omega) k_{z}-\omega}{\zeta(\omega) k_{z}+\omega} .
\end{aligned}
$$

One can generalize impedance boundary conditions to spatial dispersion case:

$$
\begin{aligned}
& \mathbf{E}_{t r}=\zeta_{r}\left(\omega, k_{r}\right)\left[\mathbf{H}_{t} \times \mathbf{n}\right]_{r}, \\
& \mathbf{E}_{t \theta}=\zeta_{\theta}\left(\omega, k_{r}\right)\left[\mathbf{H}_{t} \times \mathbf{n}\right]_{\theta} .
\end{aligned}
$$

Then one obtains two independent equations in a local basis $\mathbf{e}_{r}, \mathbf{e}_{\theta}, \mathbf{e}_{z}$ :

$$
\begin{aligned}
& N_{r} e^{i k_{z}\left(d-z_{0}\right)}+v_{1 r} e^{-i k_{z} d}=-\zeta_{r}\left(\omega, k_{r}\right)\left(-k_{r} v_{1 z} e^{-i k_{z} d}-k_{z} v_{1 r}+M_{\theta} e^{i k_{z}\left(d-z_{0}\right)}\right), \\
& N_{\theta} e^{i k_{z}\left(d-z_{0}\right)}+v_{1 \theta} e^{-i k_{z} d}=\zeta_{\theta}\left(\omega, k_{r}\right)\left(k_{z} v_{1 \theta} e^{-i k_{z} d}+M_{r} e^{i k_{z}\left(d-z_{0}\right)}\right),
\end{aligned}
$$


and reflection coefficients (162), (163) are generalized to

$$
\begin{aligned}
r_{T M 1} & =\frac{k_{z}-\zeta_{r}\left(\omega, k_{r}\right) \omega}{k_{z}+\zeta_{r}\left(\omega, k_{r}\right) \omega}, \\
r_{T E 1} & =\frac{\zeta_{\theta}\left(\omega, k_{r}\right) k_{z}-\omega}{\zeta_{\theta}\left(\omega, k_{r}\right) k_{z}+\omega} .
\end{aligned}
$$

with two functions $\zeta_{r}\left(\omega, k_{r}\right), \zeta_{\theta}\left(\omega, k_{r}\right)$. Impedance approach was first studied in the framework of the Casimir effect in Ref. [90] for evaluation of the Casimir force between two metals. In Ref. [91] the Casimir force at finite temperature is studied in impedance approach with concrete models of the function $\zeta(\omega)$ with a frequency dispersion. The high-temperature behavior of the Casimir free energy for two metals has received much attention $[92,93]$.

\section{Discussion}

In this paper, we present an explicit gauge-invariant derivation of the Casimir-Polder potential and the Casimir pressure starting from the four-current (10), (11) [54] and interaction (42).

Green functions scattering method is first applied to obtain the Casimir-Polder potential of anisotropic atom above a dielectric half-space (50) and obtain result for the Lifshitz pressure (102) in Sections 2 and 3 respectively. In Section 4 we derive the Formula (103) for the Casimir-Polder potential of anisotropic atom between two dielectric half-spaces with parallel boundaries. The result (107) [70] for the ground state energy shift of anisotropic atom between two perfectly conducting mirrors agrees with general Formula (103).

In consequent Sections the method is applied to $2+1$ conductivity layer with a frequency dispersion (Section 5), $2+1$ layer with spatial dispersion of conductivity and polarization operator of graphene (Section 6), impedance boundary conditions (Section 7), an overview of known results for these conducting systems is given.

The method developed for half-space geometries can be immediately generalized to obtain results for the Casimir-Polder potential between anisotropic atom and one or two planar plates with parallel boundaries and the Casimir pressure between two planar plates with parallel boundaries: one just must substitute reflection coefficients from the plate(s) to general Formulas (50), (103) and (102) to obtain the Casimir-Polder potential between anisotropic atom and one or two planar parallel plates and the Casimir pressure between two planar parallel plates.

Author Contributions: Investigation, both authors; writing, both authors. All authors have read and agreed to the published version of the manuscript.

Funding: This research received no external funding.

Acknowledgments: Research by V.N.M. and A.A.S. was performed at the Research park of St. Petersburg State University "Computing Center".

Conflicts of Interest: The authors declare no conflict of interest.

\section{References}

1. Casimir, H.B.G.; Polder, D. The influence of retardation on the London-van der Waals forces. Phys. Rev. 1948, 73, 360-372. [CrossRef]

2. Casimir, H.B.G. On the attraction between two perfectly conducting plates. Proc. Kon. Ned. Akad. Wet. B 1948, 51, 793-795.

3. Lifshitz, E.M. The theory of molecular attractive forces between solids. Zh. Eksp. Teor. Fiz. 1955, 29, 94-110; Translated: Sov. Phys. JETP 1956, 2, 73-83.

4. Lifshitz, E.M.; Pitaevskii, L.P. Statistical Physics, Part II; Pergamon: Oxford, UK, 1980.

5. Barash, Y.S.; Ginzburg, V.L. Electromagnetic fluctuations in matter and molecular (Van-der- Waals) forces between them. Sov. Phys. Usp. 1975, 18, 305-322. [CrossRef]

6. Barash, Y.S.; Ginzburg, V.L. Some problems in the theory of Van der Waals forces. Sov. Phys. Usp. 1984, 27, 467-491. [CrossRef]

7. Plunien, G.; Müller, B.; Greiner, W. The Casimir effect. Phys. Rept. 1986, 134, 87-193. [CrossRef]

8. Bordag, M.; Mohideen, U.; Mostepanenko, V.M. New Developments in the Casimir Effect. Phys. Rep. 2001, 353, 1-205. [CrossRef] 
9. Santangelo, E.M. Evaluation of Casimir energies through spectral functions. Theor. Math. Phys. 2002, 131, 527-542. [CrossRef]

10. Milton, K.A. The Casimir effect: recent controversies and progress. J. Phys. A Math. Gen. 2004, 37, R 209. [CrossRef]

11. Jaffe, R.L. Casimir effect and the quantum vacuum. Phys. Rev. D 2005, 72, 021301(R). [CrossRef]

12. Scheel, S; Buhmann, S.Y. Macroscopic quantum electrodynamics - concepts and applications. Acta Phys. Slovaca 2008, 58, 675-809. [CrossRef]

13. Klimchitskaya, G.L.; Mohideen, U.; Mostepanenko, V.M. The Casimir force between real materials: Experiment and theory. Rev. Mod. Phys. 2009, 81, 1827. [CrossRef]

14. Rodriguez, A.; Capasso, F.; Johnson, S. The Casimir effect in microstructured geometries. Nat. Photon 2011, 5, 211-221. [CrossRef]

15. Marachevsky, V.N. The Casimir effect: medium and geometry. J. Phys. A Math. Theor. 2012, 45, 374021. [CrossRef]

16. Woods, L.M.; Dalvit, D.A.R.; Tkatchenko, A.; Rodriguez-Lopez, P.; Rodriguez, A.W.; Podgornik, R. Materials perspective on Casimir and van der Waals interactions. Rev. Mod. Phys. 2016, 88, 045003. [CrossRef]

17. Woods, L.M.; Krüger, M; Dodonov, V.V. Perspective on Some Recent and Future Developments in Casimir Interactions. Appl. Sci. 2021, 11, 292.

18. Elizalde, E. Ten Physical Applications of Spectral Zeta Functions (Lecture Notes in Physics); Springer: Berlin/Heidelberg, Germany, 1995.

19. Kirsten, K. Spectral Functions in Mathematics and Physics; Chapman \& Hall/CRC Press: Boca Raton, FL, USA, 2002.

20. Fursaev, D.; Vassilevich, D. Operators, Geometry and Quanta: Methods of Spectral Geometry in Quantum Field Theory; Springer: Dordrecht, The Netherlands, 2011.

21. Buhmann, S.Y. Dispersion Forces; Springer: Berlin/Heidelberg, Germany, 2012; Volumes I-II.

22. Bordag, M.; Klimchitskaya, G.L.; Mohideen U.; Mostepanenko, V.M. Advances in the Casimir Effect; Oxford University Press: Oxford, UK, 2015.

23. Schram, K. On the macroscopic theory of retarded Van der Waals forces. Phys. Lett. A 1973, 43, 282-284. [CrossRef]

24. Schwinger, J. Casimir effect in source theory II. Lett. Math. Phys. 1992, 24, 59-61. [CrossRef]

25. Nesterenko, V.V.; Pirozhenko, I.G. Lifshitz formula by a spectral summation method. Phys. Rev. D 2012, 86, 052503. [CrossRef]

26. Lambrecht, A.; Marachevsky, V.N. Casimir interaction of dielectric gratings. Phys. Rev. Lett. 2008, 101, 160403. [CrossRef]

27. Lambrecht, A.; Marachevsky, V.N. Theory of the Casimir effect in one-dimensional periodic dielectric systems. Int. J. Mod. Phys. A 2009, 24, 1789-1795. [CrossRef]

28. Rayleigh, O.M. On the dynamical theory of gratings. Proc. Roy. Soc. A 1907, 79, 399-415.

29. Chan, H.B.; Bao, Y.; Zou J.; Cirelli, R.A.; Klemens, F.; Mansfield, W; Pai, C. Measurement of the Casimir force between a gold sphere and a silicon surface with nanoscale trench arrays. Phys. Rev. Lett. 2008, 101, 030401. [CrossRef]

30. Chiu, H.-C.; Klimchitskaya, G.L.; Marachevsky, V.N.; Mostepanenko, V.M.; Mohideen, U. Demonstration of the asymmetric lateral Casimir force between corrugated surfaces in the nonadditive regime. Phys. Rev. B 2009, 80, 121402(R). [CrossRef]

31. Chiu, H.-C.; Klimchitskaya, G.L.; Marachevsky, V.N.; Mostepanenko, V.M.; Mohideen, U. Lateral Casimir force between sinusoidally corrugated surfaces: Asymmetric profiles, deviations from the proximity force approximation, and comparison with exact theory. Phys. Rev. B 2010, 81, 115417. [CrossRef]

32. Derjaguin, B.V.; Abrikosova, I.I.; Lifshitz, E.M. Direct measurement of molecular attraction between solids separated by a narrow gap. Q. Rev. 1956, 10, 295-329. [CrossRef]

33. Bao, Y.; Guérout, R.; Lussange, J.; Lambrecht, A.; Cirelli, R.A.; Klemens, F.; Mansfield, W.M.; Pai, C.S.; Chan, H.B. Casimir force on a surface with shallow nanoscale corrugations: Geometry and finite conductivity effects. Phys. Rev. Lett. 2010, 105, 250402. [CrossRef]

34. Tang, L.; Wang, M.; Ng, C.Y.; Nikolic, M.; Chan, C.T.; Rodriguez, A.W.; Chan, H.B. Measurement of non-monotonic Casimir forces between silicon nanostructures. Nat. Photon 2017, 11, 97-101. [CrossRef]

35. Wang, M.; Tang, L.; Ng, C.Y.; Messina, R.; Guizal, B.; Crosse, J.A.; Antezza, M.; Chan, C.T.; Chan, H.B. Strong geometry dependence of the Casimir force between interpenetrated rectangular gratings. Nat. Commun. 2021, 12, 600. [CrossRef]

36. Antezza, M.; Chan, H.B.; Guizal, B.; Marachevsky, V.N.; Messina, R.; Wang, M. Giant Casimir torque between rotated gratings and the $\theta=0$ anomaly. Phys. Rev. Lett. 2020, 124, 013903. [CrossRef]

37. Emig, T.; Graham, N.; Jaffe, R.L.; Kardar, M. Casimir forces between arbitrary compact objects. Phys. Rev. Lett. 2007, 99, 170403. [CrossRef]

38. Rahi, S.J.; Emig, T.; Graham, N.; Jaffe, R.L.; Kardar, M. Scattering theory approach to electromagnetic Casimir forces. Phys. Rev. D 2009, 80, 085021. [CrossRef]

39. Emig, T.; Jaffe, R.L.; Kardar, M.; Scardicchio, A. Casimir interaction between a plate and a cylinder. Phys. Rev. Lett. 2006, 96, 080403. [CrossRef]

40. Canaguier-Durand, A.; Maia Neto, P.A.; Cavero-Pelaez, I.; Lambrecht, A.; Reynaud, S. Casimir Interaction between Plane and Spherical Metallic Surfaces. Phys. Rev. Lett. 2009, 102, 230404. [CrossRef]

41. Bordag, M.; Pirozhenko, I. Vacuum energy between a sphere and a plane at finite temperature. Phys. Rev. D 2010, 81, 085023. [CrossRef]

42. Markov, V.N.; Pis'mak, Y.M. Casimir effect for thin films in QED. J. Phys. A Math. Gen. 2006, 39, 6525-6532. [CrossRef]

43. Marachevsky, V.N. Casimir effect for Chern-Simons layers in the vacuum. Theor. Math. Phys. 2017, 190, 315-320. [CrossRef] 
44. Marachevsky, V.N. Casimir interaction of two dielectric half spaces with Chern-Simons boundary layers. Phys. Rev. B 2019, 99, 075420. [CrossRef]

45. Marachevsky, V.N. Chern-Simons boundary layers in the Casimir effect. Mod. Phys. Lett. A 2020, 35, 2040015. [CrossRef]

46. Fialkovsky, I.; Khusnutdinov, N.; Vassilevich, D. Quest for Casimir repulsion between Chern-Simons surfaces. Phys. Rev. B 2018, 97, 165432. [CrossRef]

47. Jiang, Q.-D.; Wilczek, F. Chiral Casimir force: Repulsive, enhanced, tunable. Phys. Rev. B 2019, 99, 125403. [CrossRef]

48. Høye, J.S.; Brevik, I. Casimir force between ideal metal plates in a chiral vacuum. Eur. Phys. J. Plus 2020, 135, 271. [CrossRef]

49. Brevik, I.; Lygren, M.; Marachevsky, V.N. Casimir-Polder effect for a perfectly conducting wedge. Ann. Phys. 1998, 267, 134-142. [CrossRef]

50. Messina, R.; Dalvit, D.A.R.; Maia Neto, P.A.; Lambrecht, A.; Reynaud, S. Dispersive interactions between atoms and nonplanar surfaces. Phys. Rev. A 2009, 80, 022119. [CrossRef]

51. Bender, H.; Stehle, C.; Zimmermann, C.; Slama, S.; Fiedler, J.; Scheel, S.; Buhmann, S.Y.; Marachevsky, V.N. Probing atom-surface interactions by diffraction of Bose-Einstein condensates. Phys. Rev. X 2014, 4, 011029. [CrossRef]

52. Levin, M.; McCauley, A.P.; Rodriguez, A.W.; Homer Reid, M.T.; Johnson S.G. Casimir repulsion between metallic objects in vacuum. Phys. Rev. Lett. 2010, 105, 090403. [CrossRef]

53. Buhmann, S.Y.; Marachevsky, V.N.; Scheel, S. Impact of anisotropy on the interaction of an atom with a one-dimensional nano-grating. Int. J. Mod. Phys. A 2016, 31, 1641029. [CrossRef]

54. Marachevsky, V.N.; Pis'mak, Y.M. Casimir-Polder effect for a plane with Chern-Simons interaction. Phys. Rev. D 2010, 81, 065005. [CrossRef]

55. Buhmann, S.Y.; Marachevsky, V.N.; Scheel, S. Charge-parity-violating effects in Casimir-Polder potentials. Phys. Rev. A 2018, 98, 022510. [CrossRef]

56. Passante, R.; Rizzuto, L.; Spagnolo, S.; Tanaka, S.; Petrosky, T.Y. Harmonic oscillator model for the atom-surface Casimir-Polder interaction energy. Phys. Rev. A 2012, 85, 062109. [CrossRef]

57. Bordag, M.; Klimchitskaya, G.L.; Mostepanenko, V.M. Nonperturbative theory of atom-surface interaction: Corrections at short separations. J. Phys. Condens. Matter 2018, 30, 055003. [CrossRef]

58. Weyl, H. Ausbreitung elektromagnetischer Wellen uber einen Leiter. Ann. Phys. 1919, 60, 481-500. [CrossRef]

59. Berestetskii, V.B.; Lifshitz, E.M.; Pitaevskii, L.P. Quantum Electrodynamics, 2nd ed.; Butterworth-Heinemann: Oxford, UK, 1982.

60. Vasiliev, A.N. Functional Methods in Quantum Field Theory and Statistics; Gordon and Breach: Amsterdam, The Netherlands, 1998.

61. Weinberg, S. The Quantum Theory of Fields; Cambridge University Press: New York, NY, USA, 1996; Volume I.

62. Khriplovich, I.B. Parity Nonconservation in Atomic Phenomena; Gordon and Breach: Philadelphia, PA, USA, 1991.

63. Tomaš, M.S. Green function for multilayers: light scattering in planar cavities. Phys. Rev. A 1995, 51, 2545-2559. [CrossRef]

64. Dzyaloshinskii, I.E.; Lifshitz, E.M.; Pitaevskii, L.P. General theory of van der Waals' forces. Sov. Phys. Usp. 1961, 4, 153-176. [CrossRef]

65. Renne, M.J. Microscopic theory of retarded van der Waals forces between macroscopic dielectric bodies. Physica 1971, 56, 125-137. [CrossRef]

66. Schwinger, J.; de Raad, L.L., Jr.; Milton, K.A. Casimir effect in dielectrics. Ann. Phys. 1978, 115, 1-23. [CrossRef]

67. Schwinger, J. Casimir energy for dielectrics. Proc. Natl. Acad. Sci. USA 1992, 89, 4091-4093. [CrossRef]

68. Tomaš, M.S. Casimir force in absorbing multilayers. Phys. Rev. A 2002, 66, 052103. [CrossRef]

69. Raabe, C.; Knöll, L.; Welsch, D.-G. Three-dimensional Casimir force between absorbing multilayer dielectrics. Phys. Rev. A 2003, 68, 033810. [CrossRef]

70. Barton, G. Quantum-electrodynamic level shifts between parallel mirrors: analysis. Proc. R. Soc. Lond. A 1987, 410, 141-174.

71. Rodriguez, A.; Ibanescu, M.; Iannuzzi, D.; Capasso, F.; Joannopoulos, J.D.; Johnson, S.G. Computation and visualization of Casimir forces in arbitrary geometries: nonmonotonic lateral-wall forces and the failure of proximity-force approximations. Phys. Rev. Lett. 2007, 99, 080401. [CrossRef] [PubMed]

72. Rahi, S.J.; Rodriguez, A.W.; Emig, T.; Jaffe, R.L.; Johnson, S.G.; Kardar, M. Nonmonotonic effects of parallel sidewalls on Casimir forces between cylinders. Phys. Rev. A 2008, 77, 030101(R). [CrossRef]

73. Emig, T. Fluctuation-induced quantum interactions between compact objects and a plane mirror. J. Stat. Mech. Theory Exp. 2008, 2008, P04007. [CrossRef]

74. Rodriguez-Lopez, P.; Rahi, S.J.; Emig, T. Three-body Casimir effects and nonmonotonic forces. Phys. Rev. A 2009, 80, 022519. [CrossRef]

75. Zaheer, S.; Rahi, S.J.; Emig, T.; Jaffe, R.L. Casimir interactions of an object inside a spherical metal shell. Phys. Rev. A 2010, 81, 030502(R). [CrossRef]

76. Rodriguez-Lopez, P.; Emig, T.; Noruzifar, E.; Zandi, R. Effect of curvature and confinement on the Casimir-Polder interaction. Phys. Rev. A 2015, 91, 012516. [CrossRef]

77. Khusnutdinov, N.; Kashapov, R.; Woods, L.M. Casimir-Polder effect for a stack of conductive planes. Phys. Rev. A 2016, $94,012513$. [CrossRef]

78. Khusnutdinov, N.; Kashapov, R.; Woods, L.M. Thermal Casimir and Casimir-Polder interactions in N parallel 2D Dirac materials. 2D Mater. 2018, 5, 035032. [CrossRef]

79. Tse, W.-K.; MacDonald, A.H. Quantized Casimir force. Phys. Rev. Lett. 2012, 109, 236806. [CrossRef] 
80. Rodriguez-Lopez, P.; Grushin, A.G. Repulsive Casimir effect with Chern insulators. Phys. Rev. Lett. 2014, 112, 056804. [CrossRef]

81. Fialkovsky, I.V.; Marachevsky, V.N.; Vassilevich, D.V. Finite-temperature Casimir effect for graphene. Phys. Rev. B 2011, 84, 035446. [CrossRef]

82. Castro Neto, A.H.; Guinea, F.; Peres, N.M.R.; Novoselov, K.S.; Geim, A.K. The electronic properties of graphene. Rev. Mod. Phys. 2009, 81, 109-162. [CrossRef]

83. Katsnelson, M.I. Graphene: Carbon in Two Dimensions; Cambridge University Press: Cambridge, UK, 2012.

84. Bordag, M.; Fialkovsky, I.V.; Gitman, D.M.; Vassilevich, D.V. Casimir interaction between a perfect conductor and graphene described by the Dirac model. Phys. Rev. B 2009, 80, 245406. [CrossRef]

85. Klimchitskaya, G.L.; Mohideen, U.; Mostepanenko, V.M. Theory of the Casimir interaction for graphene-coated substrates using the polarization tensor and comparison with experiment. Phys. Rev. B 2014, 89, 115419. [CrossRef]

86. Beneventano, C.G.; Giacconi, P.; Santangelo, E.M.; Soldati, R. Planar QED at finite temperature and density: Hall conductivity, Berry's phases and minimal conductivity of graphene. J. Phys. A Math. Theor. 2009, 42, 275401. [CrossRef]

87. Rodriguez-Lopez, P.; Kort-Kamp, W.; Dalvit, D.; Woods, L.M. Casimir force phase transitions in the graphene family. Nat. Commun. 2017, 8, 14699. [CrossRef]

88. Bordag, M.; Klimchitskaya, G.L.; Mostepanenko, V.M.; Petrov, V.M. Quantum field theoretical description for the reflectivity of graphene. Phys. Rev. D 2015, 91, 045037; Erratum in 2016, 93, 089907. [CrossRef]

89. Klimchitskaya, G.L.; Mostepanenko, V.M. Casimir and Casimir-Polder forces in graphene systems: quantum field theoretical description and thermodynamics. Universe 2020, 6, 150. [CrossRef]

90. Kats, E.I. Influence of nonlocality effects on van der Waals interaction. Zh. Eksp. Teor. Fiz. 1977, 73, $212-220$.

91. Geyer, B.; Klimchitskaya, G.L.; Mostepanenko, V.M. Surface-impedance approach solves problems with the thermal Casimir force between real metals. Phys. Rev. A 2003, 67, 062102. [CrossRef]

92. Decca, R.S.; López, D.; Fischbach, E.; Klimchitskaya, G.L.; Krause, D.E.; Mostepanenko, V.M. Precise comparison of theory and new experiment for the Casimir force leads to stronger constraints on thermal quantum effects and long-range interactions. Ann. Phys. 2005, 318, 37-80. [CrossRef]

93. Brevik, I; Ellingsen, S.E.; Milton, K.A. Thermal corrections to the Casimir effect. New J. Phys. 2006, 8, 236. [CrossRef] 